\title{
Critical Behavior of the Supersolid transition in Bose-Hubbard Models
}

\author{
Erwin Frey \\ Institut für Theoretische Physik, Physik-Department der Technischen Universität München, \\ James-Franck-Straße, D-85747 Garching, Germany \\ Leon Balents \\ Institute for Theoretical Physics, University of California, \\ Santa Barbara, CA 93106-4030
}

(September 20, 2018)

\begin{abstract}
We study the phase transitions of interacting bosons at zero temperature between superfluid (SF) and supersolid (SS) states. The latter are characterized by simultaneous off-diagonal long-range order and broken translational symmetry. The critical phenomena is described by a long-wavelength effective action, derived on symmetry grounds and verified by explicit calculation. We consider two types of supersolid ordering: checkerboard (X) and collinear (C), which are the simplest cases arising in two dimensions on a square lattice. We find that the SF-CSS transition is in the three-dimensional XY universality class. The SF-XSS transition exhibits non-trivial new critical behavior, and appears, within a $d=3-\epsilon$ expansion to be driven generically first order by fluctuations. However, within a one-loop calculation directly in $d=2$ a strong coupling fixed point with striking "non-Bose liquid" behavior is found. At special isolated multi-critical points of particle-hole symmetry, the system falls into the $3 \mathrm{~d}$ Ising universality class.

PACS numbers:67.40.Db, 05.30.Jp, $67.90+\mathrm{z}$
\end{abstract}

\section{INTRODUCTION}

Because of the dominant role played by transport measurements, conducting to insulating transitions merit special attention in condensed matter systems. In electronic systems, examples include the Anderson [1] and Mott [2] transitions [3]. In bosonic systems, interest has focused on the disorder-dominated regime: the socalled superfluid-insulator transition [⿶]. Surprisingly, the somewhat simpler problem of superfluid-insulator transitions in clean bosonic systems is less well studied. We will see that in such systems, the path from the superfluid to the insulating state generically occurs via an intermediate "supersolid" phase.

Recent work has endeavored to remedy this deficiency through numerical and mean-field studies of disorder-free interacting boson models $[5$ [12]. Such models are experimentally relevant both for helium on a periodic substrate, and for two dimensional Josephson junction arrays (see below).

\section{A. Model Hamiltonians}

In the case of Helium with a strong substrate potential, a tight-binding description is appropriate. This leads to the Bose-Hubbard Hamiltonian

$$
\begin{aligned}
H= & -t \sum_{\langle i j\rangle}\left(a_{i}^{\dagger} a_{j}+a_{j}^{\dagger} a_{i}\right)-\mu \sum_{i} n_{i} \\
& +U_{0} \sum_{i} n_{i}^{2}+U_{1} \sum_{\langle i j\rangle} n_{i} n_{j}+U_{2} \sum_{\langle\langle i k\rangle\rangle} n_{i} n_{k},
\end{aligned}
$$

where $a_{i}^{\dagger}$ and $a_{i}$ are bosonic creation and annihilation operators obeying $\left[a_{i}, a_{j}^{\dagger}\right]=\delta_{i j}$, and $n_{i}=a_{i}^{\dagger} a_{i}$. The parameter $t$ is a hopping matrix element, $\mu$ is the chemical potential, and $U_{0}, U_{1}$, and $U_{2}$ are on-site, nearest-neighbor, and next-nearest-neighbor interactions, respectively.

For a Josephson junction array, the relevant parameters are the charge $n_{i}$ residing on each superconducting island and its phase $\phi_{i}$. Including capacitive and Josephson couplings leads to the quantum phase Hamiltonian

$$
\begin{aligned}
H= & -J \sum_{\langle i j\rangle} \cos \left(\phi_{i}-\phi_{j}\right)-\mu \sum_{i} n_{i} \\
& +U_{0} \sum_{i} n_{i}^{2}+U_{1} \sum_{\langle i j\rangle} n_{i} n_{j}+U_{2} \sum_{\langle\langle i k\rangle\rangle} n_{i} n_{k},
\end{aligned}
$$

where the chemical potential is tuned by the applied gate voltage [13]. The conjugate variables satisfy the commutation relation $\left[\phi_{i}, n_{j}\right]=i \delta_{i j}$, and the strength of the Josephson phase coupling is characterized by the hopping matrix element $J$.

Finite-range interactions allow for non-trivial charge ordering with fillings of less than one particle per site. If the interactions are very strong, these are simply commensurate crystals. For weak interactions, the ground state is, by contrast, a featureless superfluid (phase ordered) state. In the intermediate regime, as discussed below, one may obtain coexisting crystallinity (charge order) and superfluidity (phase or off-diagonal-longrange-order (ODLRO)). Such a state is termed a supersolid. 


\section{B. Mean field phase diagram and Monte Carlo simulations}

The phase diagrams resulting from the above models for interacting bosons have been determined within mean-field theory $5,5,12,14,15$ and quantum Monte Carlo simulations [7 12,16 19].

A representative (mean-field) phase diagram is shown in Fig. 1, for the case of the quantum phase model with $U_{2}=0$ and $U_{1} / U_{0}=1 / 5$. The mean-field theory gives four different phases: (1) superfluid (SF), (2) Mott insulator (MI), with homogeneous density, (3) checkerboard solid (X), or Mott-insulating checkerboard charge-density wave, and (4) compressible checkerboard supersolid (XSS or SS). For $U_{2} \geq U_{1}$, "collinear" solid (C) and supersolid (CSS) phases replace the X and XSS states near halffilling 12]. The supersolid (SS) to crystal (X) transition is discussed in Refs. 20,21. It corresponds to the binding of vacancy or interstitial loops, and is expected to be mean-field like (if not first order) in two dimensions. Here, we are interested in the SS-superfluid (SF) transition, which is more analogous to a melting transition. To study it, we will employ the techniques of Landau theory and the renormalization group (RG).

\section{Results}

Our results are summarized below. The CSS-SF transition is in the $d+1$-dimensional XY universality class, with dynamical scaling exponent $z=1$. Moreover, the critical fluctuations lead to a reduction of the second sound velocity near the transition point. This reduction is, however, finite, so a conventional description of the superfluid properties applies within the critical regime.

The XSS-SF transition is more interesting. At a particular value of the chemical potential in the middle of the XSS lobe, the system has asymptotic particle-hole symmetry. At this multi-critical point, the transition is $d+1$-dimensional Ising-like $(z=1)$, and again the superfluid properties are conventional. Away from this chemical potential, however, the superfluid and critical properties become strongly coupled. Within a RG analysis in $d=3-\epsilon$ one finds a complicated crossover regime leading at low energies to "runaway flows". The absence of a stable fixed point to first order in $\varepsilon$ is often interpreted as signaling a fluctuation-driven first order transition. This conclusion must, however, be reached with some caution. Cases exist in which one finds runaway RG flows in an $\epsilon$ expansion, but the physical system (for $\epsilon$ of order one) has a continuous critical point. The most well known of these, the normal-superconducting (NS) transition in zero field 22], is actually quite similar to this problem in that the order parameter is coupled to "spectating" gapless (in the NS case, gauge) degrees of freedom.
To address this question, we apply a fixed dimension RG approach directly in $d=2$. The issue is inherently a strong-coupling one, outside of the range of applicability of a strict $\epsilon$-expansion. The fixed-dimension $R G$, while not controlled in the sense of an $\epsilon$-expansion, does allow at least an approximate treatment of the features associated with a putative "strong coupling" fixed point. In this situation, the superfluid properties are unconventional. This scenario is the bosonic analog of quantum critical modifications of electronic properties recently discussed by Millis et al. [23]. It is thereby natural to denote such anomalous superfluid behavior as "non-Bose liquid." The non-Bose liquid phase is characterized, for example, by anomalous dispersion $\omega \sim k^{z}$ for the stronglycoupled phase and CDW fluctuation modes, with $z=4 / 3$ within the fixed-dimension RG approximation.

\section{Organization}

The remainder of the paper is organized as follows. In section II we give a derivation of a field theory for the order parameter fields on the basis of general symmetry arguments. It is shown that the field theory can be transformed into a "phonon" representation, whose form is closely related to field theories describing the influence of elastic degrees of freedom on the critical behavior of magnetic systems. Section III serves to connect the correlation functions of the field theoretic model with physical observables. A short discussion of the mean-field phase diagram is given in section IV. The superfluid-collinear supersolid and superfluid-checkerboard supersolid transition are analyzed in sections V and VI using standard methods of Wilson's renormalization group theory. More sophisticated field-theoretic techniques are used in section VII to analyze the complete crossover and to calculate effective critical exponents. In the final section we give a short summary of our results and discuss some open problems.

The appendices contain additional details and explicit calculations to supplement those in the main text. A Hubbard-Stratonovich derivation of the long-wavelength effective critical action is given in Appendix A. Appendix B calculates the momentum-dependent $\Phi^{2}$ terms via an exponential cut-off scheme, needed to justify the calculation in section VI. Appendix C comprises the Feynman diagrams and the corresponding analytical expressions used in the field-theoretic RG.

\section{LANDAU THEORY}

To study the critical phenomena associated with the transition from a superfluid into a supersolid it is useful to rewrite the original Bose-Hubbard Hamiltonian for lattice bosons in terms of a field theory for the order parameter fields. A formal procedure for deriving such a 
field theory is given in appendix A. Here, we instead use general symmetry arguments to determine the most general possible action near the critical point, and hence describe the universal properties of the phase transition.

This procedure is the quantum analog of Landau's theory of thermal melting. Within this theory, Landau originally argued that such transitions must be first order. As pointed out in Ref. [21], the presence of the periodic substrate invalidates the original argument and continuous melting is in principle allowed. Here we study in detail the low-order commensurate SS states occurring near 1/2-filling, where such substrate effects are strongest.

There are two sorts of states which arise naturally in a model with up to next-nearest-neighbor interactions. If nearest-neighbor interactions dominate, the ground state is a checkerboard SS, while next-nearest-neighbor interactions favor striped (or collinear) SSs 10. The order parameters distinguishing these states are simply Fourier components of the density $n(\mathbf{x})$ :

$$
n(\mathbf{x})=n_{0}+(-1)^{\sum_{i} x_{i}} \Phi+\sum_{i}(-1)^{x_{i}} \psi_{i}
$$

Here $\Phi$ and the $\boldsymbol{\psi} \equiv\left\{\psi_{1}, \cdots, \psi_{d}\right\}$ are checkerboard and collinear order parameters, respectively. The form of the action is determined by the transformation properties of these fields. The important symmetries are translations by one lattice constant, reflections in a codimension one subspace, and $\pi / 2$ rotations within an arbitrary plane. The transformation properties of $\Phi$ and $\boldsymbol{\psi}$ under these operations are summarized in table $\mathbb{1}$. The most general local action consistent with these symmetries is

$$
\begin{aligned}
S_{0}= & \int d \mathbf{x} d \tau\left\{\frac{1}{2}\left(\frac{1}{c} \partial_{\tau} \Phi\right)^{2}+\frac{1}{2}(\nabla \Phi)^{2}+\frac{t}{2} \Phi^{2}+\frac{u}{4 !} \Phi^{4}\right. \\
& +\frac{1}{2}\left|\frac{1}{c^{\prime}} \partial_{\tau} \boldsymbol{\psi}\right|^{2}+\frac{1}{2}|\nabla \boldsymbol{\psi}|^{2}+\frac{t^{\prime}}{2}|\boldsymbol{\psi}|^{2} \\
& \left.+\frac{u^{\prime}}{4 !}|\boldsymbol{\psi}|^{4}-\frac{v^{\prime}}{4 !} \sum_{i} \psi_{i}^{4}-w \Phi \prod_{i} \psi_{i}\right\}
\end{aligned}
$$

where the coordinates and fields are rescaled such that scale of the spatial gradients is fixed to be 1 . The control parameters $t$ and $t^{\prime}$ measures the distances from the critical points, and $c$ and $c^{\prime}$ are "velocities" characterizing the crystalline order (in an incommensurate crystal phase, $c$ would be proportional to the bare phonon velocity).

Phase fluctuations. Eq. 2.2 assumes a local form of the action. Additional non-local interactions arise due to interactions with long wavelength fluctuations of the superfluid phase, $\theta$. As usual, $\theta$ is governed by the action

$$
S_{1}=\frac{\tilde{\rho}_{s}}{2 m^{2}} \int d \mathbf{x} d \tau\left\{\left(\frac{1}{v} \partial_{\tau} \theta\right)^{2}+(\nabla \theta)^{2}\right\},
$$

where $m$ is the atomic mass, and $\tilde{\rho}_{s}$ and $v$ are the "bare" superfluid density and velocity, respectively. We have taken $\tilde{\rho}_{s}$ to be a mass density, and work in units such that $\hbar=1$. The most relevant (near the critical point) coupling to the spatial order parameters allowed by the time-reversal and $U(1)$ symmetries is

$$
S_{2}=\int d \mathbf{x} d \tau\left\{i \sigma \partial_{\tau} \theta \Phi^{2}+i \sigma^{\prime} \partial_{\tau} \theta|\boldsymbol{\psi}|^{2}\right\} .
$$

Note that the factors of $i$ in Eq.(2.4) are dictated by the requirement (charge conjugation) that $S \rightarrow S^{\dagger}$ when $a \rightarrow a^{\dagger}$.

"Phonon" representation. The partition function has an alternative, purely real, representation, which is related to a classical statistical mechanical system in $d+1$ dimensions. To take advantage of insights gained in this area, we now develop an alternative representation of the phase fluctuations. For this section, we focus for simplicity on the checkerboard order, and choose $\boldsymbol{\psi}=\mathbf{0}$. Performing a Hubbard-Stratonovich transformation then results in the replacements

$$
\begin{aligned}
\frac{\tilde{\rho}_{s}}{2 m^{2}}(\nabla \theta)^{2} & \rightarrow \frac{1}{2 \tilde{\rho}_{s}} J^{2}+\frac{i}{m} \boldsymbol{J} \cdot \nabla \theta, \\
\frac{\tilde{\rho}_{s}}{2 m^{2} v^{2}}\left(\partial_{\tau} \theta\right)^{2}+i \sigma \partial_{\tau} \theta \Phi^{2} \rightarrow & \frac{v^{2}}{2 \tilde{\rho}_{s}}\left(J_{0}-m \sigma \Phi^{2}\right)^{2} \\
& +\frac{i}{m} J_{0} \partial_{\tau} \theta .
\end{aligned}
$$

The auxiliary 4-vector field $J^{\mu}$ has the physical interpretation of the Euclidean super-current. Indeed, the original phase field may now be integrated out in favor of the continuity constraint

$$
\partial_{\tau} J_{0}+\nabla \cdot \boldsymbol{J}=0
$$

Eq.(2.7) is solved by introducing the "phonon"-like field $\boldsymbol{w}$, according to

$$
\begin{aligned}
& J_{0}=-\frac{\sqrt{\tilde{\rho}_{s}}}{c} \nabla \cdot \boldsymbol{w}, \\
& \boldsymbol{J}=\frac{\sqrt{\tilde{\rho}_{s}}}{c} \partial_{\tau} \boldsymbol{w} .
\end{aligned}
$$

The final form of the action, expressed in terms of $\Phi$ and $\boldsymbol{w}$, is then

$$
\begin{aligned}
S= & \int d \mathbf{x} d \tau\left\{\frac{1}{2}\left(t \Phi^{2}+\left(\frac{1}{c} \partial_{\tau} \Phi\right)^{2}+(\nabla \Phi)^{2}\right)+\frac{\tilde{u}}{4 !} \Phi^{4}\right\} \\
& +\int d \mathbf{x} d \tau\left\{\frac{1}{2} \kappa(\nabla \cdot \mathbf{w})^{2}+\frac{1}{2}\left(\frac{1}{c} \partial_{\tau} \mathbf{w}\right)^{2}\right\} \\
& +\int d \mathbf{x} d \tau\left\{\frac{g}{2}(\nabla \cdot \mathbf{w}) \Phi^{2}\right\}
\end{aligned}
$$

where the coefficients $\tilde{u}=u+12(v / c)^{2} m^{2} c^{2} \sigma^{2} / \tilde{\rho}_{s}, \kappa=$ $(v / c)^{2}$, and $g=2(v / c)^{2} m c \sigma / \sqrt{\tilde{\rho}_{s}}$.

Closely related models have been studied in the context of the influence of elastic degrees of freedom on the behavior of magnetic systems near a critical point 
[24 26]. Depending on the number $n$ of spin components, anisotropy of the elastic constants and the symmetry of the coupling between the elastic deformations and the order parameter fields one finds different stability scenarios for the various fixed points of the renormalization group recursion relations. In the case of an isotropic coupling between the order parameter field and the phonon field the value of the specific heat exponent $\alpha$ is crucial for the character of the critical behavior. For $\alpha>0$ all fixed points are found to be unstable against anisotropic perturbations of the elastic degrees of freedom. For $\alpha<0$ the elastic degrees of freedom, isotropic as well as anisotropic, are irrelevant and the critical behavior is that of the rigid model. The effect of anisotropic couplings between the order parameter and the phonon fields has been considered in Ref. [26]. It is concluded that a first-order transition is to be expected for the Ising, anisotropic XY and Heisenberg model independent of the external conditions. In view of this earlier work it seems likely that also the above model will exhibit a topology of the flow diagram which is similar to the magnetic models and which is commonly interpreted as a signature of a fluctuation driven first-order phase transition. As we will see from the calculations in sections VVII this is actually the case. However, as will also be discussed in the following sections, this conclusion has to be reached with some care.

\section{PHYSICAL QUANTITIES AND EXPONENTS}

Near the transition point, the development of the ordered phase is reflected by the scaling behavior of the structure function. We define an Euclidean densitydensity correlation function

$$
S_{\mathrm{E}}(\mathbf{x}, \tau) \equiv\langle n(\boldsymbol{x}, \tau) n(\mathbf{0}, 0)\rangle_{C},
$$

where the subscript $C$ denotes the cumulant expectation value (connected Greens function). Substitution of Eq.(2.1) exposes the connection to the order parameters in position space:

$$
\begin{aligned}
S_{\mathrm{E}}(\boldsymbol{x}, \tau) \sim & \langle\Phi(\boldsymbol{x}, \tau) \Phi(\mathbf{0}, 0)\rangle_{C}(-1)^{\sum_{i} x_{i}} \\
& +\sum_{i j}\left\langle\psi_{i}(\boldsymbol{x}, \tau) \psi_{j}(\mathbf{0}, 0)\right\rangle_{C}(-1)^{x_{i}},
\end{aligned}
$$

where non-singular smooth contributions have been dropped. Scaling theory gives the form of these connected correlations in the critical regime,

$$
\begin{aligned}
\langle\Phi(\boldsymbol{x}, \tau) \Phi(\mathbf{0}, 0)\rangle_{C} & \sim \xi^{2-d-z-\eta} f_{ \pm}\left(x / \xi, \tau / \xi^{z}\right), \\
\left\langle\psi_{i}(\boldsymbol{x}, \tau) \psi_{j}(\mathbf{0}, 0)\right\rangle_{C} & \sim \tilde{\xi}^{2-d-\tilde{z}-\tilde{\eta}} \tilde{f}_{ \pm, i j}\left(x / \tilde{\xi}, \tau / \tilde{\xi}^{\tilde{z}}\right),
\end{aligned}
$$

where $\xi$ and $\tilde{\xi}$ are the correlation lengths for the ordering transitions of the $\Phi$ and $\boldsymbol{\psi}$ fields, respectively, $z(\tilde{z})$ and $\eta$ $(\tilde{\eta})$ are the dynamical scaling and anomalous dimension exponents, and $f_{ \pm}\left(\tilde{f}_{ \pm}\right)$are universal scaling functions (the \pm signs indicate whether the system is above or below the critical point). Additionally, there are correlation length exponents $\nu$ and $\tilde{\nu}$ which relate the correlation length to the deviation from the critical point, defined by

$$
\begin{aligned}
& \xi \sim t^{-\nu}, \\
& \tilde{\xi} \sim t^{\prime-\tilde{\nu}} .
\end{aligned}
$$

The structure function is obtained by Fourier transformation of $S_{\mathrm{E}}(\boldsymbol{x}, \tau)$. The static (equal time) correlator is thus

$$
\begin{aligned}
S_{0}(\boldsymbol{q}) \equiv & \sum_{\boldsymbol{x}} S_{\mathrm{E}}(\boldsymbol{x}, \tau=0) e^{i \boldsymbol{q} \cdot \boldsymbol{x}} \\
\sim & \xi^{2-z-\eta} g_{ \pm}[(\boldsymbol{q}-\boldsymbol{\pi}) \xi] \\
& +\sum_{i j} \tilde{\xi}^{2-\tilde{z}-\tilde{\eta}} \tilde{g}_{ \pm, i j}\left[\left(\boldsymbol{q}-\pi \hat{\boldsymbol{e}}_{i}\right) \tilde{\xi}\right]
\end{aligned}
$$

where $\boldsymbol{\pi}=(\pi, \ldots, \pi), \hat{\boldsymbol{e}}_{i}$ is a unit vector in the $x_{i}$ direction, and $g_{ \pm}$and $\tilde{g}_{ \pm, i j}$ are scaling functions. The full dynamical structure factor also has a scaling form,

$$
\begin{aligned}
S_{\mathrm{E}}(\boldsymbol{q}, \omega) \equiv & \sum_{\boldsymbol{x}} \int d \tau S_{\mathrm{E}}(\boldsymbol{x}, \tau) e^{i \boldsymbol{q} \cdot \boldsymbol{x}+i \omega \tau} \\
\sim & \xi^{2-\eta} h_{ \pm}\left[(\boldsymbol{q}-\boldsymbol{\pi}) \xi, \omega \xi^{z}\right] \\
& +\sum_{i j} \tilde{\xi}^{2-\tilde{\eta}} \tilde{h}_{ \pm, i j}\left[\left(\boldsymbol{q}-\pi \hat{\boldsymbol{e}}_{i}\right) \tilde{\xi}, \omega \tilde{\xi}^{\tilde{z}}\right]
\end{aligned}
$$

where again $h$ and $\tilde{h}$ are scaling functions (simple relations exist between $f, g$ and $h$, and may be easily derived by Fourier transformation of Eqs.(3.4)-(3.7)).

Somewhat simpler results are obtained in the isotropic, ballistic limit, when $z=1$ and $\tilde{f}_{ \pm, i j} \propto \delta_{i j}$. Focusing on the behavior near $\boldsymbol{q}=\boldsymbol{\pi}$, the dynamic structure function then takes the form

$$
S_{\mathrm{E}} \sim \xi^{2-\eta} \mathcal{S}\left[\left(|\boldsymbol{q}-\boldsymbol{\pi}|^{2} c^{2}+\omega^{2}\right) \xi^{2}\right] .
$$

A similar form holds near $\boldsymbol{q}=\hat{\boldsymbol{e}}_{i}$. At small arguments, we expect $g$ to become approximately Lorentzian. After analytic continuation $(i \omega \rightarrow \omega+i \epsilon)$, this implies the existence of a massive mode with $\omega \sim \pm \sqrt{\xi^{-2}+|\boldsymbol{q}-\boldsymbol{\pi}|^{2} c^{2}}$ for small $q$. At the critical point, regularity of $S_{\mathrm{E}}$ requires $\mathcal{S}(\chi) \sim 1 / \chi^{(2-\eta) / 2}$, and thus $S_{\mathrm{E}} \sim 1 /\left(|\boldsymbol{q}-\boldsymbol{\pi}|^{2} c^{2}+\right.$ $\left.\omega^{2}\right)^{(2-\eta) / 2}$. For $\eta>0$, this implies the behavior

$$
\operatorname{Im} S_{\text {ret. }}(\boldsymbol{q}, \omega) \sim \frac{\sin (\pi \eta / 2)}{\left(\omega^{2}-|\boldsymbol{q}-\boldsymbol{\pi}|^{2} c^{2}\right)^{1-\eta / 2}} \theta[|\omega|-|\boldsymbol{q}-\boldsymbol{\pi}| c],
$$

and thus a continuum of modes with linear dispersion.

In addition to these quantities, the critical fluctuations of the checkerboard order parameter modify the behavior of the superfluid. To study these modifications, it is useful to define the current-current correlation function 


$$
D_{i j}(\boldsymbol{x}, \tau) \equiv\left\langle J^{i}(\boldsymbol{x}, \tau) J^{j}(\mathbf{0}, 0)\right\rangle,
$$

where $\boldsymbol{J}=\left(\tilde{\rho}_{s} / m\right) \boldsymbol{\nabla} \theta$ is the super-current. By introducing an infinitesimal generating field into Eqs.(2.2)-(2.4) and differentiating the final effective action twice, one easily obtains the expression

$$
D_{i j}(\boldsymbol{x}, \tau)=\tilde{\rho}_{s}\left[\frac{1}{c^{2}}\left\langle\partial_{\tau} w^{i}(\boldsymbol{x}, \tau) \partial_{\tau} w^{j}(\mathbf{0}, 0)\right\rangle-\delta^{i j}\right]
$$

for $D$ in the phonon representation.

The superfluid density $\rho_{s}$ and second-sound velocity $v_{s}$ may be extracted from the long wavelength form

$$
D_{i j}(\boldsymbol{k}, \omega) \sim \rho_{s} \frac{k_{i} k_{j}}{k^{2}+\omega^{2} / v_{s}^{2}},
$$

for $k, \omega \rightarrow 0$.

\section{MEAN FIELD THEORY}

Eq.(2.2) exhibits four phases in mean-field theory. In the superfluid (SF) phase there is no spatial order, so $\Phi=0$ and $\boldsymbol{\psi}=\mathbf{0}$. The checkerboard (X) state has $\phi \neq 0$ but $\boldsymbol{\psi}=\mathbf{0}$. A collinear (C) or striped phase occurs with $\Phi=0$ but $\boldsymbol{\psi} \propto \hat{\boldsymbol{e}}_{i}$. Lastly, there is a mixed (M) phase in which no symmetries persist, so $\Phi \neq 0$ and $\boldsymbol{\psi} \neq \mathbf{0}$ and does not point along a symmetry axis of the lattice.

We have been unable to determine the detailed geometry of the mean-field phase diagram. However, we expect the general topology shown in Fig.2. For large $t$ and $t^{\prime}=0$, there is a critical line separating the SF and $\mathrm{C}$ phases, while for large $t^{\prime}$ and $t=0$, a different phase boundary separates the SF and X states.

\section{SUPERFLUID-COLLINEAR SS TRANSITION}

For large $t$, the checkerboard order parameter $\Phi$ is strongly suppressed and plays no role in the SF-C transition. We therefore consider here Eq.(2.2) with $\Phi=0$ and thereby neglect $w$ (indeed, integrating out the massive $\Phi$ field leads only to a small renormalization of $u^{\prime}$ and $v^{\prime}$ ). For concreteness, we now also focus directly on the case $d=2$, in which $\boldsymbol{\psi}$ is a two-component field.

In the absence of coupling to the superfluid phase mode (i.e. $\sigma^{\prime}=0$ ), the action is identical to the classical free energy of an XY ferromagnet with cubic (square) anisotropy $v^{\prime}$ in $D=2+1=3$ dimensions. For positive $v^{\prime}$ (as assumed here), the collinear state is favored for $t^{\prime}<0$ in mean-field theory. To study the critical properties for $D=3$, however, we must include the effects of fluctuations.

Such fluctuations have been studied (with $\sigma^{\prime}=0$ ) by many authors [27 35]. There is a general agreement between epsilon expansion and direct RG calculations as well as experimental observations [35] that cubic anisotropy is in fact an irrelevant perturbation on the $\mathrm{XY}$ fixed point in three dimensions, with RG eigenvalue $-1.8 \lesssim \lambda_{4} \lesssim-1.4$. Therefore, the SF-C transition with $\sigma^{\prime}=0$ should be XY-like, i.e. $\tilde{\nu} \approx .67, \tilde{\eta} \approx 0.040$, and $\tilde{z} \approx 2$. Note, however, that $v^{\prime}$ is in fact a dangerous irrelevant operator, because it is necessary to select the direction of $\boldsymbol{\psi}$ in the ordered phase [36].

This asymptotic restoration of the $U(1)$ symmetry should lead to the emergence of a "pseudo-Goldstone" mode near the critical point in the ordered phase. To see this, consider following the RG flows (for $t^{\prime}<0$ ) until the renormalized reduced temperature is of order one. At this point, the rescaled $\boldsymbol{\psi}$ will have amplitude of order one (enforced by the negative $|\psi|^{2}$ and positive $|\psi|^{4}$ terms), but almost unconstrained angle $\Theta \equiv \tan ^{-1}\left(\psi_{2} / \psi_{1}\right)$. The renormalized action for $\Theta$ is then of the form

$$
\begin{aligned}
S_{\mathrm{R}, \Theta}= & \tilde{\xi}^{-3} \int d^{2} \boldsymbol{x} d \tau\left\{\frac{1}{2} \tilde{\xi}^{2}\left[\left(\frac{1}{c^{\prime}} \partial_{\tau} \Theta\right)^{2}+(\nabla \Theta)^{2}\right]\right. \\
& \left.-v^{\prime} \tilde{\xi}^{\lambda_{4}}\left[\cos ^{4} \Theta+\sin ^{4} \Theta\right]\right\} .
\end{aligned}
$$

Expanding the cosine and sine terms in $\Theta$ gives the dispersion $\omega^{2}=p^{2} c^{\prime 2}+\Delta_{\perp}^{2}$, with the anomalously small gap $\Delta_{\perp}^{2}=4 v^{\prime} \tilde{\xi}^{-\left(2+\left|\lambda_{4}\right|\right)}$. Note that the longitudinal gap $\Delta_{\|} \propto \overline{\tilde{\xi}}^{-1}$ is much larger than $\Delta_{\perp}$. The system appears isotropic in the sense that $\Delta_{\perp} / \Delta_{\|} \rightarrow 0$ as $\tilde{\xi} \rightarrow \infty$. A detailed study of the effect of cubic anisotropy on the critical behavior below $T_{c}$ can be found in Ref. 37].

Until now we have neglected the interaction with the phase $\theta$. Remarkably, the XY fixed point is stable against these fluctuations. As described in detail in Ref. [21], the relevance of $\sigma^{\prime}$ at the "classical" critical point is determined by the specific heat exponent $\tilde{\alpha}=2-D \tilde{\nu}$. In particular, the eigenvalue $\lambda_{\sigma}=\tilde{\alpha} /(2 \tilde{\nu})$ is slightly negative at the three dimensional XY fixed point, and the long wavelength physics at the critical point is just that of a decoupled $D=3 \mathrm{XY}$ critical point and a free massless boson (phase) field.

Another way to understand this result is to integrate out the density fluctuations $\boldsymbol{\psi}$ and determine their effect upon the superfluid modes. This is easily accomplished perturbatively in $\sigma^{\prime}$, since the coupling is precisely to the energy operator $|\psi|^{2}$. At order $\sigma^{\prime}$, one produces only a boundary $\left(\int i \partial_{\tau} \theta\right)$ contribution. The first non-trivial term occurs at $O\left(\sigma^{\prime 2}\right)$, where one finds a contribution to the effective action

$$
\begin{aligned}
\Delta S_{1} & =\frac{{\sigma^{\prime}}^{2}}{2} \int_{1,2} \partial_{\tau} \theta_{1} \partial_{\tau} \theta_{2}\left\langle|\psi|_{1}^{2}|\psi|_{2}^{2}\right\rangle_{C} \\
& \sim{\sigma^{\prime}}^{\prime 2} \tilde{\xi}^{2(1-\tilde{\alpha}) / \tilde{\nu}} \int_{1,2} \partial_{\tau} \theta_{1} \partial_{\tau} \theta_{2} \mathcal{C}\left[\left|\boldsymbol{x}_{1}-\boldsymbol{x}_{2}\right| / \tilde{\xi},\left|\tau_{1}-\tau_{2}\right| / \tilde{\xi}\right] \\
& \sim{\sigma^{\prime}}^{\prime} \tilde{\xi}^{\tilde{\alpha} / \tilde{\nu}} \int_{\boldsymbol{x}, \tau}\left|\partial_{\tau} \theta\right|^{2}
\end{aligned}
$$

where the subscripts 1,2 are introduced as a shorthand notation for the integration variables $\boldsymbol{x}_{1}, \tau_{1}$ and $\boldsymbol{x}_{2}, \tau_{2}, \mathcal{C}$ 
is a (short-range) scaling function describing the energyenergy correlations, and the last line is obtained via a gradient expansion of $\theta_{2}$. The final correction term may be interpreted as a renormalization of the second sound velocity,

$$
v_{\mathrm{R}}^{\prime 2}=\frac{v^{\prime 2}}{1+C{\sigma^{\prime}}^{2} m^{2} \xi^{\tilde{\alpha} / \tilde{\nu}} v^{\prime 2} / \tilde{\rho}_{s}},
$$

where $C$ is an order one constant. Note that for $\tilde{\alpha}<0$, as is the case here, this is a small reduction to the velocity near the critical point. For $\tilde{\alpha}>0$, however, the correction diverges, and the $v^{\prime}$ appears to be driven to zero! This will be the case for the SF-X transition discussed in the next section and requires a more careful analysis. Here we simply point out that the second sound velocity undergoes a finite suppression by critical fluctuations (note that, once $\tilde{\alpha}$ is negative, non-singular contributions actually dominate over the singular one exhibited explicitly in Eq.(5.3), and lead to a finite correction to $v_{\mathrm{R}}^{\prime}$ ).

\section{SUPERFLUID-CHECKERBOARD SUPERSOLID TRANSITION}

In this section we discuss the situation when $t^{\prime} \gg 1, u^{\prime}$, but $t$ passes through zero. In this case it is the $\boldsymbol{\psi}$ modes which are weakly fluctuating, and the resulting theory is simply that of the $\Phi$ field coupled to the superfluid phase. Here the critical behavior for $\sigma=0$ is simply that of a classical three-dimensional Ising model. However, because the specific heat exponent in this case is positive ( $\alpha \approx 0.12$ ), these phase fluctuations are relevant, and the problem needs to be reconsidered.

To do so, we employ the technique of $\varepsilon$-expansion, developing $\mathrm{RG}$ equations near the upper critical dimension $D_{\mathrm{uc}}=d_{\mathrm{uc}}+1=4$. In this section, we proceed with the simplest such method, known as a momentum-shell RG, which is sufficient to extract most aspects of the critical behavior. In section VII, we use more powerful field theoretic methods within dimensional regularization to compute full crossover quantities. Here the theory is regularized by including a sharp cut-off in momenta: all the functional integrals in the partition function $Z$ are only over fields with momenta $|q|<\Lambda$, the cut-off wavevector. Such a cut-off serves as a crude approximation to the physical lattice cut-off of the bosonic system, but the precise form is irrelevant for the perturbative RG to the order required here, and facilitates the calculation of certain quantities.

The momentum shell RG is performed by successive elimination of shells of momenta near the cut-off $\Lambda$. After each shell is integrated out of the partition function, the coordinates and momenta are rescaled to keep the value of the cut-off fixed. Additional rescalings of the fields allows most of the quadratic part of the action to remain fixed as well. The remaining coefficients flow under the RG. While an infinite number of operators are generated, only the relevant (and marginal) ones need be kept within the $\varepsilon$ expansion, in this case $u, \sigma$, and $v$.

Momentum shell RG suffers, unfortunately, from one flaw: it is unable to deal properly with situations in which loop integrals must be handled at non-zero external momenta (and frequencies). For most problems, such momentum and frequency dependence can indeed be neglected to $O(\varepsilon)$ in the RG. In a cubic theory such as this one, however, the second diagram in Fig. 13 can potentially produce corrections at $O(\varepsilon)$, because the coupling $\sigma$ is only $O\left(\varepsilon^{1 / 2}\right)$. We investigate this diagram in appendix $\mathrm{B}$, and find that these dangerous contributions are actually not present. The momentum shell RG is therefore well defined to leading order in $\varepsilon$, and we proceed here with the remainder of the calculation.

We define the mode elimination step of the RG by integrating out fields with momenta in the range $\Lambda / b<$ $p<\Lambda$, and use the critical dynamics method of integrating out all frequencies, which are not cut off. By choosing $b=e^{-d l}$, where $d l$ is infinitesimal, we arrive at differential RG flow equations for the coupling constants. These equations are easily computed diagrammatically. Because we evaluate all loops at zero external momenta (c.f. the previous paragraph), the constraint that both loop momenta be within the shell is trivially satisfied by constraining just the single integration variable, due to momentum conservation.

Consider first the diagram in Fig. 14. Because of the explicit imaginary time derivative in the coupling $\sigma$, this diagram generates a renormalization of the velocity $v$. Because of the explicit factor of $i$ in the vertex, it decreases $v$ as in the perturbative treatment of the previous section. Because this is the only renormalization of $v$ at one loop, the search for possible critical fixed points must proceed in the limit $v \rightarrow 0$. This simplifies the computation of certain diagrams. In particular, any internal $\theta$ lines may be treated in this limit as simply constant $v^{2}$ factors. All remaining loop integrals are then proportional to the standard form

$$
\int_{\Lambda / b<p<\Lambda} \frac{d^{3} \boldsymbol{p}}{(2 \pi)^{3}} \int_{-\infty}^{\infty} \frac{d \omega}{2 \pi} \frac{1}{\left(p^{2}+\omega^{2} / c^{2}\right)^{2}}=A_{3} c d l / 4
$$

where $A_{3}=1 /\left(2 \pi^{2}\right)$ is a geometric factor. To complete the calculation, the fields and coordinates are rescaled to keep the cut-off fixed, according to

$$
\begin{aligned}
x \rightarrow b x, & \tau \rightarrow b^{z} \tau, \\
p \rightarrow p / b, & \omega \rightarrow \omega / b^{z}, \\
\Phi \rightarrow \Phi b^{(2-d-z-\eta) / 2}, & \theta \rightarrow \theta b^{(2-d-z-\tilde{\eta}) / 2},
\end{aligned}
$$

where in this case, because of the absence of momentumdependent loop corrections, $\eta=\tilde{\eta}=z-2=0$ (to this order). The flow equations resulting from the rescaling and mode elimination are 


$$
\begin{aligned}
\frac{d K}{d \ell} & =(1-U / 4-5 K / 2) K, \\
\frac{d U}{d \ell} & =U-\left(3 U^{2} / 8+6 K U+24 K^{2}\right), \\
\frac{d \tilde{V}}{d \ell} & =-K V / 4, \\
\frac{d\left(t-t_{0}\right)}{d l} & =[2-\varepsilon(U / 8+K)]\left(t-t_{0}\right),
\end{aligned}
$$

where we have defined $\varepsilon=3-d, \ell=\varepsilon l$, and the dimensionless couplings $K=A_{3} \sigma^{2} c v^{2} / \varepsilon, U=A_{3} u c / \varepsilon$, and $V=v / c$. The $T_{c}$ shift $t_{0}=-\varepsilon \Lambda^{2}(U / 8+K)$. The RG calculation is controlled for $d=3-\varepsilon$, where the coupling constants are of order $\varepsilon$, and higher loop corrections to the flow equations are higher order in $\varepsilon$. Note that $K$ is strictly positive, so $V$ indeed flows monotonically to zero.

The flow diagram resulting from Eqs. (6.5)-(6.6) is shown in Fig. 3. The three-dimensional classical Ising fixed point $(U=8 / 3, K=0)$ is unstable once phase fluctuations are introduced. However, a new weak-coupling fixed point is not found within a one-loop $\varepsilon$-expansion. Instead, the couplings flow into the classically unstable regime where $U<0$. This scenario is generally interpreted as a fluctuation-induced first order phase transition 38]. As was mentioned in the introduction, the existence of a strong coupling fixed point can not be excluded within the above line of argument. In fact, upon using a field theoretic RG approach carried out in fixed dimension, we will show in the next section that a strong coupling fixed point can be identified for large enough values of the coupling constant $V$. The resulting "non-Bose liquid" behavior will be discussed in the next section.

For small values of $V$ "runaway trajectories" persist. In that case the instabilities in the trajectories may indicate a fluctuation-driven first order transition may actually describe the physics of the supersolid transition. One can understand this by matching the RG flows onto a renormalized classical theory. Close enough to the critical point (the flows may be integrated down to the scale of the correlation length), the renormalized theory has a negative value of $u$. Mean-field theory is justified for the renormalized action, since the effective $t$ is now order one. With a negative $u$, a standard mean-field analysis then predicts a first order transition before the critical point is actually reached. Note that, if the initial coupling $\sigma$ (and hence $K$ ) is very small, this will require an extremely close approach to the critical point, and hence result in extremely small discontinuities in thermodynamic parameters (such as $\langle\Phi\rangle$ ). Experiments or numerical simulations which do not probe sufficiently close to $t_{0}$ may not resolve the true first-order transition, and instead observe "effective" exponents (or more complex cross-over behavior). At a true fixed point, the correlation length exponent would be determined from Eq. 6.8) as $1 / \nu=2-\varepsilon(U / 8+K)$. A rough estimate of the measured value of $\nu$ may be obtained from an examination of Fig. 3. Using values of $U$ and $K$ along a flow from the (unstable) Ising fixed along the central trajectory in the figure suggests a gradual reduction of $\nu_{\text {eff. }}$ from approximately $0.6(\nu=0.6$ is the $O(\varepsilon)$ result for the Ising value - the accepted value is $\nu \approx 0.64$ ) towards 0.5 .

\section{PERTURBATION THEORY AND RENORMALIZATION: DIMENSIONAL REGULARIZATION}

In this section we use methods adapted from field theory to investigate the critical behavior of the superfluid to checkerboard supersolid transition. This allows us to go beyond the results obtained in section $\mathrm{VI}$ and study the full crossover behavior near criticality. Furthermore, we can study the flow of the parameters and coupling constants for arbitrary values of the second sound velocity. This will turn out to be an important technical advantage, since it allows us to identify a strong coupling fixed point above a critical value of $v / c$ (see below).

A perturbation theory for the effective free energy functional of the supersolid phase may be set up following the common procedure (see e.g. Ref. [38]). The elements of perturbation theory are the free propagators for the one-component density order parameter $\Phi$

$$
G_{\Phi \Phi}(\mathbf{k}, \omega)=\frac{1}{t_{0}+\left(\omega / c_{0}\right)^{2}+k^{2}},
$$

and the superfluid phase

$$
G_{\Theta \Theta}(\mathbf{k}, \omega)=\frac{1}{\left(\omega / v_{0}\right)^{2}+k^{2}},
$$

and the vertices, which may be read off from the anharmonic parts of Eqs. (2.2)-(2.4). In Fig. 1 we depict these elements for constructing the Feynman graphs of the field-theoretical representation of the critical behavior of a supersolid. Following the standard notation in field theory bare values are indicated by a subscript " 0 ".

\section{A. Renormalization factors}

For $t_{0} \rightarrow 0$ the perturbation theory is infrareddivergent leading to non-trivial critical exponents. These anomalous dimensions are derived by studying the ultraviolet singularities of the field theory, which appear at the upper critical dimension $d_{c}=3$, when the momentum cutoff $\Lambda$ is pushed to infinity (see e.g. Ref. [38]). Finite values are then assigned to these UV-divergent integrals through the application of a regularization prescription. We shall choose the dimensional regularization scheme as introduced by t'Hooft and Veltman [39]; here the $(\Lambda \rightarrow \infty)$ singularities appear as poles $\propto 1 /\left(d_{c}-d\right)$.

The ultraviolet divergences may then be collected in renormalization constants and absorbed into the definition of multiplicatively renormalized quantities. Thus we define the renormalized fields 


$$
\Phi=Z_{\Phi}^{1 / 2} \Phi_{0} \quad, \quad \Theta=Z_{\Theta}^{1 / 2} \Theta_{0},
$$

and the renormalized parameters

$$
\begin{aligned}
t & =Z_{\Phi}^{-1} Z_{t}\left(t_{0}-t_{0 c}\right) \mu^{-2}, \\
c^{2} & =Z_{\Phi} Z_{c}^{-1} c_{0}^{2}, \\
v^{2} & =Z_{\Phi} Z_{v}^{-1} v_{0}^{2}, \\
u & =Z_{\Phi}^{-2} Z_{u} u_{0} \mu^{d-3} S_{d}, \\
\sigma & =Z_{\Theta}^{-1 / 2} Z_{\Phi}^{-1} Z_{\sigma} \sigma_{0} \mu^{(d-3) / 2} S_{d}^{1 / 2} .
\end{aligned}
$$

In Eq. (7.4) we have taken into account the fact that the fluctuations will also shift the transition temperature. Furthermore we have rendered the renormalized quantities dimensionless by introducing the explicit arbitrary length scale $1 / \mu$, and have finally included the geometric factor

$$
S_{d}=\frac{2}{(4 \pi)^{d / 2} \Gamma(d / 2)} .
$$

Adapting a fixed dimension $R G$ approach, one finds from the one-loop expressions for the singular parts of the vertex functions $\Gamma_{02}, \Gamma_{20}, \Gamma_{12}$, and $\Gamma_{04}$ in appendix $\bar{Q}$ the following results for the renormalization constants:

$$
\begin{aligned}
& Z_{t}=1-\frac{u c}{8 \varepsilon}-\frac{(v \sigma)^{2} c}{\varepsilon} \frac{1}{(1+v / c)^{2}}, \\
& Z_{\Phi}=1-\frac{(v \sigma)^{2} c}{\varepsilon} \frac{v / c}{(1+v / c)^{3}} \mathcal{A}(v / c, d), \\
& Z_{c}=1+\frac{2(v \sigma)^{2} c}{\varepsilon} \frac{v / c}{(1+v / c)^{3}}, \\
& Z_{v}=1+\frac{(v \sigma)^{2} c}{2 \varepsilon}, \\
& Z_{u}=1-\frac{3 u c}{8 \varepsilon}-\frac{6(v \sigma)^{2} c}{\varepsilon} \frac{1}{(1+v / c)^{2}} \\
&-\frac{24(v \sigma)^{4} c^{2}}{\varepsilon u c} \frac{1}{(1+v / c)^{3}}, \\
& Z_{\sigma}= 1-\frac{u c}{4 \varepsilon}-\frac{2(v \sigma)^{2} c}{\varepsilon} \frac{1}{(1+v / c)^{2}} .
\end{aligned}
$$

For the fixed-dimension $\mathrm{RG}$, the quantity $\mathcal{A}$ depends on both $V$ and $d$ (see Eq. (C7) in appendix C). As a consequence of the momentum dependence of the three-point vertex, there are no singular contributions to the $q^{2}$-term in the harmonic part of the superfluid phase field to any order in perturbation theory. This implies the exact identity

$$
Z_{\Theta}=1
$$

\section{B. Renormalization group equations and flow functions}

The renormalization group equation serves to connect the asymptotic theory, where the infrared singularities manifest themselves, with a region in parameter space where the couplings $u$ and $\sigma$ are finite (but not necessarily small), and an ordinary "naive" perturbation expansion becomes applicable. It explicitly takes advantage of the scale invariance of the system near the critical point. More precisely, we observe that the bare two-point vertex function is of course independent of the arbitrary renormalization scale $\mu$ :

$$
\left.\mu \frac{d}{d \mu}\right|_{0} \Gamma_{l n}^{0}\left(r_{0}, c_{0}, v_{0}, u_{0}, \sigma_{0}, \mathbf{q}, \omega\right)=0 .
$$

Introducing Wilson's flow functions

$$
\begin{aligned}
\zeta_{\Phi} & =\mathcal{D}_{\mu} \ln Z_{\Phi}, \quad \zeta_{\Theta}=\mathcal{D}_{\mu} \ln Z_{\Theta}, \\
\zeta_{t} & =\mathcal{D}_{\mu} \ln \frac{t}{t_{0}-t_{0 c}}=-2-\zeta_{\Phi}+\mathcal{D}_{\mu} \ln Z_{t}, \\
\zeta_{c} & =\mathcal{D}_{\mu} \ln \frac{c}{c_{0}}=\frac{1}{2} \zeta_{\Phi}-\frac{1}{2} \mathcal{D}_{\mu} \ln Z_{c}, \\
\zeta_{v} & =\mathcal{D}_{\mu} \ln \frac{v}{v_{0}}=\frac{1}{2} \zeta_{\Theta}-\frac{1}{2} \mathcal{D}_{\mu} \ln Z_{v}, \\
\zeta_{\sigma} & =\mathcal{D}_{\mu} \ln \frac{\sigma}{\sigma_{0}}=\frac{d-3}{2}-\frac{\zeta_{\Theta}}{2}-\zeta_{\Phi}+\mathcal{D}_{\mu} \ln Z_{\sigma}, \\
\zeta_{u} & =\mathcal{D}_{\mu} \ln \frac{u}{u_{0}}=(d-3)-2 \zeta_{\Phi}+\mathcal{D}_{\mu} \ln Z_{u},
\end{aligned}
$$

where $\mathcal{D}_{\mu}=\left.\mu \frac{\partial}{\partial \mu}\right|_{0}$ denotes a logarithmic derivative with fixed bare couplings and parameters, Eq. (7.17) leads to renormalization group equations for the renormalized vertex functions $\Gamma_{l n}$. For instance, the two-point vertex function of the Ising fields $\Phi$ satisfies the partial differential equation

$$
\begin{aligned}
& {\left[\mu \frac{\partial}{\partial \mu}+\zeta_{t} t \frac{\partial}{\partial r}+\zeta_{c} c \frac{\partial}{\partial c}+\zeta_{v} v \frac{\partial}{\partial v}+\zeta_{\sigma} \sigma \frac{\partial}{\partial \sigma}\right.} \\
& \left.+\zeta_{u} u \frac{\partial}{\partial u}+\zeta_{\Phi}\right] \Gamma_{02}(\mu, t, c, v, u, \sigma, \mathbf{k}, \omega)=0 .
\end{aligned}
$$

The RG equation (7.24) is now readily solved with the method of characteristics. The characteristics $a(s)$ of Eq. (7.24) define the running parameters and coupling constants into which these transform when $\mu \rightarrow \mu(s)=$ $\mu s$. They are given by the solutions to first-order differential equations $(a=t, c, v, u, \sigma)$

$$
s \frac{d a(s)}{d s}=\zeta_{a}(s) a(s),
$$

with the initial conditions $t(1)=t, c(1)=c, v(1)=v$, $u(1)=u$, and $\sigma(1)=\sigma$, namely

$$
a(s)=a \exp \left[\int_{1}^{s} \zeta_{a}\left(s^{\prime}\right) d s^{\prime} / s^{\prime}\right] .
$$

Defining the dimensionless vertex function $\hat{\Gamma}_{02}$ according to 


$$
\Gamma_{02}(\mu, t, c, v, u, \sigma, \mathbf{k}, \omega)=\mu^{2} \hat{\Gamma}_{02}\left(t, u, \sigma, \frac{v}{c}, \frac{\mathbf{k}}{\mu}, \frac{\omega^{2}}{c^{2} \mu^{2}}\right)
$$

the solution of Eq. (7.24) is

$$
\begin{aligned}
& \Gamma_{02}(\mu, t, c, v, u, \sigma, \mathbf{k}, \omega)=\mu^{2} s^{2} \exp \left[\int_{1}^{s} \zeta_{\Phi}\left(s^{\prime}\right) d s^{\prime} / s^{\prime}\right] \\
& \times \hat{\Gamma}_{02}\left(t(s), u(s), \sigma(s), \frac{v(s)}{c(s)}, \frac{\mathbf{k}}{\mu s}, \frac{\omega^{2}}{c(s)^{2} \mu^{2} s^{2}}\right) .
\end{aligned}
$$

\section{Flow diagram and fixed points}

To one-loop order Wilson's flow functions as derived from Eqs. (7.18)-(7.23) read

$$
\begin{aligned}
\zeta_{\Phi} & =(v \sigma)^{2} c \frac{v / c}{(1+v / c)^{3}} \mathcal{A}(v / c, d) \\
\zeta_{c} & =\frac{1}{2} \zeta_{\Phi}+(v \sigma)^{2} c \frac{v / c}{(1+v / c)^{3}} \\
\zeta_{v} & =\frac{1}{2} \zeta_{\Theta}+\frac{1}{4}(v \sigma)^{2} c \\
\zeta_{t} & =-2-\zeta_{\Phi}+\frac{1}{8} u c+(v \sigma)^{2} c \frac{1}{(1+v / c)^{2}}, \\
\zeta_{\sigma} & =\frac{d-3}{2}-\frac{\zeta_{\Theta}}{2}-\zeta_{\Phi}+\frac{1}{8} u c+(v \sigma)^{2} c \frac{1}{(1+v / c)^{2}} \\
\zeta_{u} & =(d-3)-2 \zeta_{\Phi}+\frac{3}{8} u c+6(v \sigma)^{2} c \frac{1}{(1+v / c)^{2}} \\
& +24 \frac{(v \sigma)^{4} c^{2}}{u c} \frac{1}{(1+v / c)^{3}}
\end{aligned}
$$

and due to the exact relation Eq. (7.16) one finds

$$
\zeta_{\Theta}=0 .
$$

The zeros of the $\beta$-functions,

$$
\begin{aligned}
& \beta_{u}=\left.\mu \frac{\partial}{\partial \mu}\right|_{0} u=u \zeta_{u}, \\
& \beta_{\sigma}=\left.\mu \frac{\partial}{\partial \mu}\right|_{0} \sigma=\sigma \zeta_{\sigma},
\end{aligned}
$$

determine the values of the coupling constants where the theory becomes scale invariant.

In order to study the flow diagram, it is convenient to introduce new effective coupling constants $K:=\sigma^{2} v^{2} c$, $U:=u c$, and $V:=v / c$, whose flow equations can be readily obtained from Eqs. 7.18)-(7.23). They are

$$
\begin{aligned}
\frac{1}{K} \frac{d K}{d \ell} & =\varepsilon-\frac{U}{4}-K\left[\frac{2}{(1+V)^{2}}+\frac{1}{2}+\frac{V(1-3 \mathcal{A} / 2)}{(1+V)^{3}}\right], \\
\frac{1}{U} \frac{d U}{d \ell} & =\varepsilon-\frac{3}{8} U-24 \frac{K^{2}}{U(1+V)^{3}}
\end{aligned}
$$

$$
\begin{array}{r}
-K\left[\frac{6}{(1+V)^{2}}+\frac{V(1-3 \mathcal{A} / 2)}{(1+V)^{3}}\right], \\
\frac{1}{V} \frac{d V}{d \ell}=-K\left[\frac{1}{4}-\frac{V(1+\mathcal{A} / 2)}{(1+V)^{3}}\right]
\end{array}
$$

where, for the purpose of comparison with Eqs. 6.5 6.7) we have defined the running logarithmic scale $\ell=-\ln s$, so that $d / d \ell=-s d / d s$. Note that $\mathcal{A}$ depends on $V$ and $d$ (see Eq. (C7) in appendix Q1). Depending on the sign of the expression in the square brackets of Eq. (7.40), $\mathcal{B}(V, d)=1 / 4-V(1+\mathcal{A}(V, d) / 2) /(1+V)^{3}$, the flow of $V$ either tends to $V=0$ or to $V=\infty$. One finds that there is a critical value $V_{c}(d)$, where $\mathcal{B}(V, d)$ changes sign. One of the most essential features of $V_{c}(d)$ is that it cannot be obtained within a strict $\varepsilon$-expansion. As can be inferred

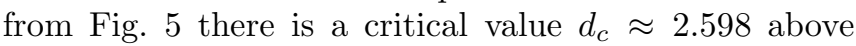
which there is no solution of the equation $\mathcal{B}(V, d)=0$. For $d<d_{c}$ there are two real roots, where one diverges upon approaching $d=2$. Since we are primarily interested in the critical behavior in $d=2$ dimensions the most relevant root is given by the lower curve in Fig. 5 . In what follows we will restrict our discussion to the case $d=2$. Then the flow-equations have the following fixed points

$$
\begin{array}{llll}
V=0, & K=0, & U=0 & \text { (Gaussian I) } \\
V=0, & K=0, & U=8 / 3 & \text { (Ising I) } \\
V=\infty, & K=0, & U=0 & \text { (Gaussian II) } \\
V=\infty, & K=0, & U=8 / 3 & \text { (Ising II) } \\
V=\infty, & K=4 / 3, & U=16 / 3 & \text { (Non-Bose liquid). }
\end{array}
$$

The crucial quantity for the behavior of the coupling constants is the ratio, $V=v / c$, of the velocity of the second sound $v$ and phonon velocity $c$ (first sound). For $V<V_{c}^{1}(d=2) \equiv V_{c}$ it turns out that the flow of $V$ tends to zero, in which case both the Gaussian I and the Ising I fixed points are unstable to small perturbations. This corresponds to the runaway trajectories discussed in the preceding section. However, the behavior of the renormalization group trajectories become quite different for $V>V_{c}$. Then the flow of $V$ tends to infinity, but it still depends on the sign of the coefficients in the flow equations for $U$ and $K$, whether the renormalization group trajectories show runaway flow or converge into a finite fixed point. In the limit $V \rightarrow \infty$ the flow equations reduce to the following simple form

$$
\begin{gathered}
\frac{1}{K} \frac{d K}{d \ell}=1-\frac{1}{4} U+\frac{1}{4} K, \\
\frac{1}{U} \frac{d U}{d \ell}=1-\frac{3}{8} U+\frac{3}{4} K,
\end{gathered}
$$

the flow diagram of which is shown in Fig. 6 .

The manifold, which separates the region in parameter space, where the flow of the renormalization group trajectories ends up in the non-Bose liquid fixed point (stable trajectory), from the parameter space where the 
flow shows runaway behavior (unstable trajectory), is called the separatrix. A visualization of the complex flow-behavior is given in Fig. 7, where we have chosen the renormalization group trajectories to start at the plane $V=3$ (bottom of the box). This part of the parameter space shows stable as well as unstable trajectories, where all stable trajectories are attracted to a critical surface, which finally contracts to the non-Bose liquid fixed point. A numerical analysis of the flow equations also shows that approximately below $V=2$ there are no more stable trajectories. In Figs. 8 and 9 two-dimensional projections of the flow diagram are shown with starting values $V=3$ and $V=1$, respectively.

\section{Scaling behavior and effective exponents}

Now we turn to the investigation of Eq. (7.28) near a fixed point $\mathbf{G}^{\star}=\left(K^{\star}, U^{\star}, V^{\star}\right)$; introducing the fixedpoint values of the Wilson flow functions $\zeta_{a}^{\star}=\zeta_{a}\left(\mathbf{G}^{\star}\right)$, also called the anomalous dimension of the parameter $a$, one finds the following scaling law

$$
\begin{aligned}
& \Gamma_{02}(\mu, t, \mathbf{G}, \mathbf{k}, \omega)=\mu^{2} s^{2+\zeta_{\Phi}^{\star}} \\
& \quad \times \hat{\Gamma}_{02}\left(t s^{\zeta_{t}^{\star}}, \mathbf{G}^{\star}, \frac{\mathbf{k}}{\mu s}, \frac{\omega^{2}}{c^{2} \mu^{2} s^{2+2 \zeta_{c}^{\star}}}\right) .
\end{aligned}
$$

For $\Gamma_{20}$ one obtains a similar scaling law, where $\zeta_{\Phi}$ and $\zeta_{c}$ are replaced by $\zeta_{\Theta}$ and $\zeta_{v}$, respectively. Using the matching condition $k / \mu s=1$ the following dynamic scaling law,

$$
\begin{aligned}
& \Gamma_{02}(\mu, t, \mathbf{G}, \mathbf{k}, \omega) \propto q^{2-\eta_{\Phi}} \\
& \quad \times \hat{\Gamma}_{02}\left(\frac{t}{(k / \mu)^{1 / \nu}}, \mathbf{G}^{\star}, 1, \frac{\omega^{2}}{\mu^{2} c^{2}(k / \mu)^{2 z}}\right),
\end{aligned}
$$

is obtained, where the five independent exponents

$$
\begin{aligned}
\eta_{\Theta} & =-\zeta_{\Theta}^{\star}=0, \\
z_{\Theta} & =1+\zeta_{v}^{\star}, \\
\eta_{\Phi} & =-\zeta_{\Phi}^{\star}, \\
z_{\Phi} & =1+\zeta_{c}^{\star}, \\
\nu & =-\frac{1}{\zeta_{t}^{\star}}, \\
\Delta & =\zeta_{v}^{\star}-\zeta_{c}^{\star} .
\end{aligned}
$$

All other exponents are found through the scaling relations $\gamma=\nu(2-\eta)$ and $2 \beta+\gamma=2-\alpha=\beta(1+\delta)$.

The crossover exponent $\Delta$ describes the flow of the ratio of the two sound velocities, $V(s)=v(s) / c(s) \rightarrow$ $s^{\Delta} v / c$. As discussed in the previous section there is a separatrix in parameter space which separates stable from unstable trajectories. In the unstable region all trajectories show runaway behavior, but depending on the initial value of $V$ being smaller or larger than $V_{c}$ the flow of $V$ either tends to zero or to infinity.
Let us consider two extreme situations: For $V=0$ we find $\beta_{K}=-K+\frac{5}{2} K^{2}+\frac{1}{4} K U$ and $\beta_{U}=-U+\frac{3}{8} U^{2}+$ $6 K U+24 K^{2}$ with the corresponding fixed points:

$$
\begin{aligned}
& K=0, \quad U=0 \quad(\text { Gaussian I), } \\
& K=0, \quad U=\frac{8}{3} \quad(\operatorname{Ising} \mathrm{I}) \text {. }
\end{aligned}
$$

The fixed point values for the flow-functions at the Ising fixed point are $\zeta_{\Phi}^{\star}=0, \zeta_{c}^{\star}=0, \zeta_{v}^{\star}=0$, and $\zeta_{t}^{\star}=-2+\frac{1}{3}$. Hence we find the following critical exponents at the Ising fixed point (to one-loop order):

$$
\begin{aligned}
\eta_{\Phi} & =0 \\
z_{\Theta}=z_{\Phi} & =1 \\
\nu & =\frac{1}{2}+\frac{1}{6} .
\end{aligned}
$$

For $V=\infty$ we find in $d=2$ dimensions $\beta_{K}=-K-$ $\frac{1}{4} K^{2}+\frac{1}{4} K U$ and $\beta_{U}=-U+\frac{3}{8} U^{2}-\frac{3}{4} K U$ with the corresponding fixed points:

$$
\begin{array}{lll}
K=0, & U=0 & \\
K=0, & U=8 / 3 & (\text { Ising II }) \\
K=4 / 3, & U=16 / 3 & \text { (Non-Bose liquid). }
\end{array}
$$

The fixed point values of the flow-functions at the nonBose liquid fixed point $\operatorname{read} \zeta_{\Phi}^{\star}=2 / 3, \zeta_{c}^{\star}=1 / 3$, $\zeta_{v}^{\star}=1 / 3, \zeta_{t}^{\star}=-2$ and $\zeta_{\sigma}=1 / 2$, where interestingly all fluctuation contributions to the renormalization of $t$ cancel. Hence we find the following critical exponents at the non-Bose liquid fixed point (to one-loop order):

$$
\begin{aligned}
\eta_{\Phi} & =-\frac{2}{3}, \\
z_{\Phi} & =\frac{4}{3}, \\
z_{\Theta} & =\frac{4}{3}, \\
\nu & =\frac{1}{2} .
\end{aligned}
$$

Now, we consider crossover effects on the most interesting physical quantities, namely the correlation functions of the density order parameter and the superfluid phase. For the discussion we will use an approximation, frequently called renormalized mean field theory, where one uses the fully renormalized flow-functions, but the zero-loop result for the scaling function. Thus one gets

$$
\begin{gathered}
\Gamma_{\Theta \Theta}=\Gamma_{20}(t, \mathbf{q})=\mu^{2} s^{2} \exp \left[\int_{1}^{s} \zeta_{\Theta}\left(s^{\prime}\right) d s^{\prime} / s^{\prime}\right] \\
\times\left[\frac{k^{2}}{\mu^{2} s^{2}}+\frac{\omega^{2}}{\mu^{2} s^{2} v^{2}(s)}\right] \\
\Gamma_{\Phi \Phi}=\Gamma_{02}(t, \mathbf{q})=\mu^{2} s^{2} \exp \left[\int_{1}^{s} \zeta_{\Phi}\left(s^{\prime}\right) d s^{\prime} / s^{\prime}\right] \\
\times\left[t(s)+\frac{k^{2}}{\mu^{2} s^{2}}+\frac{\omega^{2}}{\mu^{2} s^{2} c^{2}(s)}\right] .
\end{gathered}
$$


The most convenient way to analyze the crossover behavior of the correlation functions is in terms of effective critical exponents. We consider first the case $t=0$ and choose to define the effective dynamic critical exponents

$$
\begin{aligned}
& z_{\Theta}(s)=1+\zeta_{v}(s), \\
& z_{\Phi}(s)=1+\zeta_{c}(s),
\end{aligned}
$$

which are shown in Fig. 10 as a function of the flow parameter $s$, where for the initial values we have chosen $U(s=1)=8 / 3, K(s=1)=1 / 100$ and a series of ratios of sound velocities $V(1)=10,5,3$. Upon using the matching condition $q / \mu s=1$ the flow parameter $s$ can be related to the wave number. A corresponding effective Fisher exponent for the Ising field can also be defined by $\eta_{\Phi}=-\zeta_{\Phi}(s)$. The crossover behavior is shown in Fig. 11 for the same set of initial values as for the dynamic critical exponents.

At zero external frequencies and momenta one can define an effective critical exponent for the dependence of the Ising field correlation function on the control parameter

$$
v(s)=-1 / \zeta_{t}(s) .
$$

Using again the same set of initial values for the coupling constants one obtains the crossover behavior depicted in Fig. 12 .

\section{SUMMARY AND CONCLUSIONS}

We have studied the SS-SF critical phenomena using renormalization group techniques. Physical results are obtained by extrapolating from $d=3-\epsilon$ to $d=2$, corresponding to bosons on a periodic substrate. Coupling to the gapless phase modes of the superfluid opens the possibility of unusual quantum-critical behavior. This is not realized for the CSS-SF transition, in which the coupling of critical and phase modes is irrelevant, and the universality class is simply three-dimensional XY-like. By contrast, the mode-coupling terms are relevant for the XSS-SF transition, and generically modify the critical behavior. The $\epsilon$ expansion predicts a fluctuation-driven first order transition in this case. A fixed dimension RG, however, gives a different scenario. For small values of the ratio $V=v / c$ runaway trajectories persist indicating again a first order transition though we cannot rule out the possibility of a strong-coupling continuous critical point. For large values of $V$ we have identified a strong coupling fixed point. If the latter occurs, the phase and critical density modes must be strongly coupled at the transition, resulting in anomalous superfluid behavior. At the non-Bose liquid fixed point the exponent for the correlation length is found to be $\nu=1 / 2$ to one loop order, since all fluctuation corrections cancel. It would be interesting to check whether there are twoloop contributions to this classical value. The value for the Fisher exponent $\eta_{\Phi}=-2 / 3$ is negative, similar as in the case of a normal to superconducting transition [22]. In either case, there are special multi-critical points in the phase diagram (at particular values of the chemical potential) for which the mode-coupling terms explicitly vanish by particle-hole symmetry, and simple $3 \mathrm{~d}$ Ising behavior (and ordinary superfluidity) is restored.

An attempt to extract critical properties at the XSS$\mathrm{SF}$ transition in the quantum phase model has been made in Ref. 12]. In this model the multi-critical point may be exactly located at $n_{0}=1 / 2$ using particle-hole symmetry. There, they find a correlation exponent $\nu=0.55 \pm 0.05$. This is somewhat less than the expected 3d Ising behavior $\nu \approx 0.64$. They also quote a value $z=1$ here, in agreement with the RG. We believe the disagreement with the $3 \mathrm{~d}$ Ising result at the particle-hole symmetric point (where the theory appears to be on extremely firm ground) is probably due to finite-size corrections in the numerical data. Away from this point (they study $n_{0}=0.4$ ), the correlation length exponent is perhaps reduced, $\nu=0.5 \pm 0.11$, although the larger error bars make this interpretation difficult. A reduction of $\nu$ from $\nu \approx 0.64 \rightarrow 0.5$ is indeed predicted by the crossover analysis for a scenario, where a non-Bose liquid fixed point exists. In the latter case the dynamic critical exponent is found to be $z=4 / 3$ to one-loop order. Interestingly, scaling analysis of their Monte-Carlo data does not appear to differentiate between a dynamical scaling exponent $z=1$ or $z=2$. However, they put a stronger constraint on the combination $z+\eta=0.8 \pm 0.2$, which is consistent with $z+\eta=4 / 3-2 / 3 \approx 0.67$. This gives some support to the $\mathrm{RG}$ prediction, though further systematic analysis of the simulation data is certainly desirable.

More recently, Scalettar et al. 40] have investigated the tendency to phase separation in the Bose-Hubbard model as a more sensitive indicator for a possible first order transition. Their preliminary results appear to confirm the existence of a first order transition in the XSS$\mathrm{SF}$ case, but not for the CSS-SF transition. This is in agreement with our RG results, which predict that the CSS-SF transition remains continuous (3d XY-like) even away from the multi-critical point.

An interesting open problem is to address the critical behavior in the XSS-SF case directly in two dimensions more closely, to ascertain whether a fluctuation-driven first order transition or a continuous transition obtains in that case for small values of $V$. This might either be achieved by a two-loop renormalization group calculation accompanied by some resummation technique or by a self-consistent calculation, which appears to capture much of the physics in the mathematically similar problem of the normal-superconducting transition in zero field [22]. Whether an analogous dual formulation of the problem can yield some insight is presently unclear. 


\section{ACKNOWLEDGMENTS}

It is a pleasure to acknowledge helpful discussions with Anne van Otterlo. We would also like to thank Jan Wilhelm for helping us with generating some of the figures. The work of E.F. has been supported by the Deutsche Forschungsgemeinschaft (DFG) under contract no. Fr $850 / 2$ and no. SFB 266. L.B.'s work was supported at the Institute for Theoretical Physics by grant no. PHY94-07194. We gratefully acknowledge financial support through EU Contract ERBCHRX-CT920020 during a workshop at the Institute for Scientific Interchange (ISI) in Torino, where part of this work has been done. 


\section{APPENDIX A: MEAN-FIELD DECOUPLING}

The long-wavelength action Eq. (2.2) can be derived using the method of auxiliary fields. Because the supersolid phase exhibits two broken symmetries, it is necessary to introduce two such fields, which will become order parameters for ODLRO and crystallinity. For concreteness, and because the most likely experimental realization of the SS is in Josephson junction arrays, we work here with the quantum phase model; the BoseHubbard Hamiltonian may be treated by similar methods. For simplicity, we also focus here on the case with only nearest-neighbor interactions, in which case $\boldsymbol{\psi}$ ordering does not arise. Standard techniques can be used to translate the Hamiltonian Eq. (1.2) into the Euclidean action

$$
\begin{aligned}
S_{\mathrm{E}}= & \int d \tau\left\{\sum_{i}\left(i n_{i} \partial_{\tau} \phi_{i}-\mu n_{i}\right)\right. \\
& \left.-J \sum_{\langle i j\rangle} \cos \left(\phi_{i}-\phi_{j}\right)+\sum_{i j} \frac{1}{2} U_{i j} n_{i} n_{j}\right\},
\end{aligned}
$$

where $U_{i j}=U_{0} \delta_{i j}+U_{1} \delta_{|i-j|, 1}$. The action enters the functional integral formulation of quantum mechanics via the generating functional (partition function)

$$
Z=\int[d n][d \phi] e^{-S_{\mathrm{E}}}
$$

The Josephson interaction may be decoupled using the (Hubbard-Stratonovich) identity

$$
\begin{aligned}
& \exp \left[\int d \tau \sum_{\langle i j\rangle} \cos \left(\phi_{i}-\phi_{j}\right)\right] \\
& =\int[d \bar{\psi}][d \psi] \exp \left[-\int d \tau\left\{\psi_{i} e^{-i \phi_{i}}+\bar{\psi}_{i} e^{i \phi_{i}}\right.\right. \\
& \left.\left.\quad+\left(T^{-1}\right)_{i j} \bar{\psi}_{i} \psi_{j}\right\}\right]
\end{aligned}
$$

where the index sums on the right hand side are implied (we will adopt this convention in the remainder of this appendix), and the matrix $T_{i j} \equiv J \delta_{|i-j|, 1}$. As is usually the case with such decouplings, the functional integral over the $\psi$ 's is best defined by a product measure in momentum space. To obtain convergence, the contour for $\tilde{T}(\boldsymbol{q})<0$ is then taken along the imaginary axis. Before decoupling the charge interaction, we first separate out the zero momentum component, according to

$$
U_{i j}=W_{i j}+\bar{U} \delta_{i j}
$$

where $\bar{U}=U_{0}+2 d U_{1}$. In momentum space, the remaining coupling is strictly negative,

$$
\tilde{W}(\mathbf{q} \neq 0)=-2 d U_{0}+2 U_{0} \sum_{a=1}^{d} \cos q_{a}<0 .
$$

We may therefore perform a second HubbardStratonovich to replace

$$
\frac{1}{2} W_{i j} n_{i} n_{j} \rightarrow \Phi_{i} \epsilon_{i} n_{i}-\frac{1}{2}\left(W^{-1}\right)_{i j} \epsilon_{i} \Phi_{i} \epsilon_{j} \Phi_{j} .
$$

We choose to define the auxiliary field with a factor of $\epsilon_{i}=(-1)^{\sum_{a} x_{a}}$ to bring out the checkerboard correlations. Note that because $\tilde{W}<0$, the functional integral over $\Phi$ is well behaved (the theory is properly defined by omitting the zero mode, since $\tilde{W}(\mathbf{0})=0)$. The last sum can be rewritten

$$
-\frac{1}{2}\left(W^{-1}\right)_{i j} \epsilon_{i} \Phi_{i} \epsilon_{j} \Phi_{j}=\int_{\boldsymbol{q}}-\frac{1}{2}|\tilde{\Phi}(\boldsymbol{q})|^{2} / \tilde{W}(\boldsymbol{q}+\boldsymbol{\pi})
$$

where $\tilde{W}(\boldsymbol{q}+\boldsymbol{\pi})=-2 d U_{1}-2 U_{1} \sum_{a=1}^{d} \cos q_{a} \approx-4 d U_{1}[1-$ $\left.q^{2} /(4 d)\right]$ for small $q$. Combining results, we obtain the full effective action

$$
\begin{aligned}
S_{\mathrm{eff}}= & \int d \tau \frac{d^{d} \boldsymbol{q}}{(2 \pi)^{d}}\left\{\frac{1}{8 d U_{1}}\left(1+\frac{q^{2}}{4 d}\right)|\tilde{\Phi}|^{2}\right. \\
& \left.+\frac{1}{2 J d}\left(1+\frac{q^{2}}{2 d}\right)|\tilde{\psi}|^{2}\right\}+\sum_{i} s_{1 i},
\end{aligned}
$$

where we have expanded $\tilde{T}$ around $\boldsymbol{q}=\mathbf{0}$, and introduced the single-particle action

$$
\begin{aligned}
s_{1 i}= & \int d \tau\left\{i n_{i} \partial_{\tau} \phi_{i}-\alpha_{i} \cos \left(\phi_{i}-\theta_{i}\right)\right. \\
& \left.+\frac{1}{2} \bar{U} n_{i}^{2}-\mu n_{i}+\Phi_{i} \epsilon_{i} n_{i}\right\},
\end{aligned}
$$

with $\psi_{i}=-\alpha_{i} e^{i \theta_{i}} / 2$. It is useful to first perform the shift $\phi_{i} \rightarrow \phi_{i}+\theta_{i}$, which removes the $\theta$ dependence of the cosine at the cost of a (time-dependent) shift of the chemical potential, $\mu \rightarrow \tilde{\mu}_{i}=\mu-i \partial_{\tau} \theta_{i}$. Integration over the $\phi_{i}$ and $n_{i}$ variables can then formally be performed independently. It is exactly equivalent to the solution of decoupled quantum-rotator models, each of which has the single-particle Hamiltonian

$$
H_{1}=\frac{\bar{U}}{2} n^{2}-\tilde{\mu} n+\Phi \epsilon n-\alpha \cos \phi .
$$

Eq. A10 should be supplemented by the commutation relation $[\phi, n]=i$. We will assume slow variations of the phase, so that $\partial_{\tau} \theta_{i}$ is approximately constant.

The mean-field theory of Refs. [6, 9] is recovered in the saddle point approximation, $\delta S_{\text {eff }} / \delta \bar{\psi}=\delta S_{\text {eff }} / \delta \Phi=0$. This gives the conditions

$$
\begin{aligned}
& \alpha_{i}=4 d J\left\langle e^{i \phi_{i}}\right\rangle, \\
& \Phi_{i}=-4 d U_{1} \epsilon_{i}\left\langle n_{i}\right\rangle .
\end{aligned}
$$

Because we wish to obtain a Landau theory, allowing for the possibility of fluctuations, we will explicitly construct the effective action $S_{\text {eff }}$ as a function of $\Phi$ and the 
Goldstone mode $\theta$. It is sufficient to work perturbatively in $\Phi$. This is accomplished via the cumulant expansion

$$
s_{1, \mathrm{eff}}=\sum_{k} \frac{(-1)^{k+1}}{k !} \int_{\tau_{1} \cdots \tau_{k}}\left\langle n\left(\tau_{1}\right) \cdots n\left(\tau_{k}\right)\right\rangle_{C} \Phi_{1} \cdots \Phi_{k} \epsilon^{k},
$$

where the (cumulant) expectation value is evaluated using the single-site theory with $\Phi=0$. For constant $\Phi$, the sum in Eq. (A13) is just the imaginary time integral of the ground state energy, $\int d \tau E_{1}(\Phi)$.

Unfortunately, even these expectation values cannot be evaluated exactly, due to the form of Eq. (A10). As discussed in Ref. [6], the one-site Hamiltonian may be rewritten in the phase representation as that of a particle in a cosine potential. The associated Schrödinger equation is therefore a Mathieu equation, whose solutions are not available in closed form. We therefore concentrate on the region $n_{0} \equiv \mu / \bar{U}=1 / 2+\delta$, with $\delta \ll 1$, in which the checkerboard phase emerges most strongly, and analytic progress is possible.

The one site problem may be recast in dimensionless form, using

$$
\tilde{H}_{1} \equiv H_{1} / \bar{U}=n^{2} / 2-(1 / 2+\lambda) n-\beta\left(a+a^{\dagger}\right),
$$

where $\lambda=\delta-\left(i \partial_{\tau} \theta+\epsilon \Phi\right) / \bar{U}, \beta=\alpha /(2 \bar{U})$, and $a=e^{i \phi}$. The operators $a$ and $a^{\dagger}$ obey the usual creation and annihilation operator commutation relations with respect to the number operator, $[a, n]=a$, but commute among themselves $\left[a, a^{\dagger}\right]=0$. This allows the existence of states with arbitrarily (positive or negative) integer number, and one should note that $n \neq a^{\dagger} a$.

For small $\delta$, and $\beta=0$, there are two nearly degenerate low energy states: $|0\rangle$, with $n=0$ and energy $\tilde{E}_{1}(n=0)=0$, and $|1\rangle$, with $n=1$ and energy $\tilde{E}_{1}(n=1)=-\lambda$ (since we are eventually going to expand in $\partial_{\tau} \theta$ and $\Phi, \lambda$ is essentially equal to $\delta$. All other states (with $n \neq 0,1$ ) have the much larger energies $\tilde{E}_{1}(n \neq 0,1)$ of order one. For $\beta \ll 1$, we may therefore work within the restricted basis of $n=0,1$, in which the Hamiltonian is represented

$$
\tilde{H}_{1}=-\lambda / 2+\lambda / 2 \sigma^{z}-\beta \sigma^{x},
$$

where $\boldsymbol{\sigma}$ is the vector of Pauli matrices in the standard representation. The energy eigenvalues for $\beta \neq 0$ in this approximation are easily obtained,

$$
\tilde{E}_{1 \pm}=(-\lambda \pm \Delta) / 2
$$

where the gap $\Delta=\sqrt{\lambda^{2}+4 \beta^{2}}$. The two corresponding (normalized) eigenstates are

$$
\begin{aligned}
& |-\rangle=\frac{1}{\sqrt{2 \Delta}}\left(\sqrt{\Delta-\lambda}|0\rangle+\frac{2 \beta}{\sqrt{\Delta-\lambda}}|1\rangle\right), \\
& |+\rangle=\frac{1}{\sqrt{2 \Delta}}\left(-\sqrt{\Delta+\lambda}|0\rangle+\frac{2 \beta}{\sqrt{\Delta+\lambda}}|1\rangle\right) .
\end{aligned}
$$

The value of $\beta$ is fixed by the mean-field condition, Eq. (A11), equivalent to minimizing the effective Hamiltonian density

$$
\mathcal{H}_{\beta}=\frac{\bar{U}}{2}\left[-\delta-\Delta_{0}+\frac{\bar{U}}{J d} \beta^{2}\right]
$$

where $\Delta_{0}=\sqrt{\delta^{2}+4 \beta^{2}}$, and we have taken $\lambda=\delta$. Corrections to $\lambda$ will be included via the Landau expansion. The minimum occurs when $\beta=(4 d / \bar{U}) \sqrt{J^{2}-J_{c}^{2}}$, for $J>J_{c}=\delta \bar{U} /(8 d)$, and $\beta=0$ for $J<J_{c}$. For $\delta=0$, $J_{c}$ vanishes, because an arbitrarily weak hopping matrix element breaks the degeneracy between empty and singly occupied sites.

We are now ready to derive the Landau expansion of the effective action. The Hubbard-Stratonovich measure Eq. (A\&) gives

$$
\begin{aligned}
S_{\text {eff }, 1}= & \int d^{d} \boldsymbol{x} d \tau\left\{\frac{1}{8 d U_{1}} \Phi^{2}+\frac{1}{32 d^{2} U_{1}}|\nabla \Phi|^{2}\right. \\
& \left.+\frac{\bar{U}^{2} \beta^{2}}{4 d^{2} J}|\nabla \theta|^{2}\right\},
\end{aligned}
$$

where we neglected amplitude fluctuations of $\psi$. A second contribution is obtained simply by expanding the one-site ground-state energy in $\Phi$ and $\partial_{\tau} \theta$,

$$
\begin{aligned}
\Delta S_{\mathrm{eff}, 2}= & \int d \tau \sum_{i}-\frac{\bar{U}}{2} \Delta_{i} \\
& =\int d^{d} \boldsymbol{x} d \tau\left\{-\frac{\beta^{2}}{\bar{U} \Delta_{0}^{3}} \Phi^{2}+\frac{\beta^{2}\left(\beta^{2}-\delta^{2}\right)}{\bar{U}^{3} \Delta_{0}^{7}} \Phi^{4}\right. \\
& \left.+\frac{1}{\bar{U} \Delta_{0}^{3}}\left(\partial_{\tau} \theta\right)^{2}-\frac{3 \beta^{2} \delta}{\bar{U}^{2} \Delta_{0}^{5}} i \partial_{\tau} \theta \Phi^{2}\right\}
\end{aligned}
$$

up to constants. The only remaining non-trivial term is $\left(\partial_{\tau} \Phi\right)^{2}$. It cannot be calculated from the ground state energy $E_{1}$, because it arises from the time dependence in $H_{1}$, for which stationary quantum mechanics is not valid. It can, however, be calculated from the more general cumulant expansion, Eq. (A13), which also provides a check on the $\Phi^{2}$ coefficient in Eq. (A21). Taking $k=2$ in Eq. (A13) gives

$$
\begin{aligned}
\Delta S_{\mathrm{eff}}= & -\frac{1}{2} \sum_{i} \int_{\tau, \tau^{\prime}}\left\langle n(\tau) n\left(\tau^{\prime}\right)\right\rangle_{C} \Phi_{i}(\tau) \Phi_{i}\left(\tau^{\prime}\right) \\
& =-\int \frac{d \omega}{2 \pi} \frac{C(\omega)}{2}|\Phi(\omega)|^{2},
\end{aligned}
$$

where we used time translation invariance to express the result in terms of $C(\omega) \equiv \int d \tau\langle n(\tau) n(0)\rangle_{C} e^{i \omega \tau}$. The imaginary time correlation function is easily expressed quantum mechanically as

$$
\begin{aligned}
C(\tau)= & \left\langle-\left|n e^{-\left(H-E_{1-}\right)|\tau|} n\right|-\right\rangle-\langle-|n|-\rangle^{2} \\
& =\frac{\beta^{2}}{\Delta_{0}^{2}} e^{-\bar{U} \Delta|\tau|}
\end{aligned}
$$


after inserting the complete set (in this approximation) $|+\rangle\langle+|+|-\rangle\langle-|$, and using Eqs. A16 A18. Fourier transforming gives $C(\omega)=\left(2 \beta^{2} / \Delta_{0}\right)\left[U /\left(U^{2} \Delta_{0}^{2}+\omega^{2}\right)\right]$. For $\omega=0$, Eq. (A22) then agrees with the $\Phi^{2}$ term calculated earlier, and provides the "kinetic" correction

$$
\Delta S_{\mathrm{eff}, 3}=\int d^{d} \boldsymbol{x} d \tau \frac{\beta^{2}}{\bar{U}^{3} \Delta_{0}^{5}}\left|\partial_{\tau} \Phi\right|^{2} .
$$

The full effective action is thus $S_{\mathrm{eff}}=S_{\mathrm{eff}, 1}+S_{\mathrm{eff}, 2}+$ $S_{\text {eff }, 3}$. It is brought into the standard form of Eqs. 2.2 2.4 by the rescaling

$$
\Phi \rightarrow 4 d \sqrt{U_{1}} \Phi
$$

After rescaling, the Landau parameters are

$$
\begin{aligned}
t & =4 d-\frac{32 d^{2} \beta^{2} U_{1}}{\Delta_{0}^{3} \bar{U}}, \\
u & =6144 d^{4} \frac{\beta^{2}\left(\beta^{2}-\delta^{2}\right)}{\Delta_{0}^{7}} \frac{U_{1}^{2}}{\bar{U}^{3}}, \\
c^{2} & =\frac{\Delta_{0}^{5}}{32 d^{2} \beta^{2}} \frac{\bar{U}^{3}}{U_{1}}, \\
v^{2} & =\frac{\beta^{2} \Delta_{0}^{3}}{4 d^{2}} \frac{\bar{U}^{3}}{J}, \\
\sigma & =-\frac{48 d^{2} \beta^{2} \delta}{\Delta_{0}^{5}} \frac{U_{1}}{\bar{U}^{2}}, \\
\frac{\rho_{s}}{m^{2}} & =\frac{\beta^{2}}{2 d^{2}} \frac{\bar{U}^{2}}{J} .
\end{aligned}
$$

From these equations, we can determine the bare velocity ratio $V=v / c$, as

$$
V^{2}=32 d^{2}\left(\frac{J}{\bar{U}}\right) \frac{U_{1}}{\bar{U}}
$$

at the multi-critical point $\delta=0$. These results are valid for $\beta \sim J / \bar{U} \ll 1$, so that $V \ll 1$ (since $U_{1}<\bar{U}$ ). In this limit the $V \ll 1$ approximation used in section $\mathrm{VI}$ is valid even in the early stages of renormalization (i.e. uniformly at all scales).

\section{APPENDIX B: LACK OF MOMENTUM-DEPENDENT LOOP CONTRIBUTION TO $\Phi$ PROPAGATOR}

In this appendix we consider the integral

$$
I(k, \omega) \equiv \int_{\boldsymbol{p}, \nu} \frac{\nu^{2}}{p^{2}+\nu^{2} / v^{2}} \frac{e^{-|p| a-|\boldsymbol{p}+\boldsymbol{k}| a}}{(\boldsymbol{p}+\boldsymbol{k})^{2}+(\nu+\omega)^{2}},
$$

where $\int_{\boldsymbol{p}, \nu} \equiv \int \frac{d^{3} \boldsymbol{p}}{(2 \pi)^{3}} \frac{d \nu}{2 \pi}$, and $a=1 / \Lambda$. We have taken $d=3$ to obtain the leading term of $O\left(\varepsilon^{0}\right)$, chosen an exponential cut-off for momenta, and set $c=1$. We will also make use of the limit $v \rightarrow 0$, to which the theory is driven (within the $\varepsilon$-expansion) by the monotonic negative velocity renormalization. It is crucial here that both internal lines are cut-off: physically, we define the theory unambiguously by confining all momenta of the theory to be less than $\Lambda$. In the momentum shell RG, we would perform an analogous integral iteratively in order to reduce $\Lambda$. Here we will instead perform the entire integral with fixed cut-off. It may then be differentiated with respect to $\Lambda$ to yield the appropriate contribution from a particular scale. Any renormalizations must thus appear as logarithmic divergences, i.e. $\ln (\Lambda / k)$ factors in the integral. In particular, the coefficients of $k^{2} \ln (\Lambda / k)$ and $\omega^{2} \ln (\Lambda / k)$ determine the renormalization of the $|\nabla \Phi|^{2}$ and $\left(\partial_{\tau} \Phi\right)^{2}$ terms in the action, respectively.

Focusing first on the gradient term, consider $I_{0}(k) \equiv$ $I(k, \omega=0)$. The frequency integral may be simply performed by contour integration to yield

$$
I_{0}(k)=\frac{v^{2}}{2} \int_{\boldsymbol{p}_{1}, \boldsymbol{p}_{2}} \frac{1}{p_{2}+v p_{1}} e^{-p_{1} a-p_{2} a}(2 \pi)^{3} \delta\left(\boldsymbol{p}_{1}+\boldsymbol{p}_{2}+\boldsymbol{k}\right),
$$

where we have inserted a delta function to put the momenta of the two propagators on equal footing. Using the Fourier representation $(2 \pi)^{3} \delta(\boldsymbol{k})=\int_{\boldsymbol{x}} \exp [i \boldsymbol{k} \cdot \boldsymbol{x}]$ and going to angular coordinates $y=\cos (\theta)$, where $\theta$ is the azimuthal angle, we have

$$
\begin{aligned}
I_{0}= & \frac{v^{2}}{2} \int_{\boldsymbol{x}} \frac{e^{i \boldsymbol{k} \cdot \boldsymbol{x}}}{(2 \pi)^{4}} \int^{\Lambda} d p_{1} d p_{2}\left(p_{1} p_{2}\right)^{2} \int_{-1}^{1} d y_{1} d y_{2} \\
& \frac{1}{p_{2}+v p_{1}} e^{i x\left(p_{1} y_{1}+p_{2} y_{2}\right)}
\end{aligned}
$$

where $\int^{\Lambda} d p \equiv \int_{0}^{\Lambda} d p e^{-p a}$. Performing the $y$-integrals gives

$$
I_{0}=\frac{v^{2}}{2 \pi^{3} k} \int_{0}^{\infty} d x \frac{\sin k x}{x} \int^{\Lambda} d p_{1} d p_{2} p_{1} p_{2} \frac{\sin p_{1} x \sin p_{2} x}{p_{2}+v p_{1}}
$$

The $v \rightarrow 0$ limits of the $p$-integrals are well defined. Taking it gives

$$
I_{0}=\frac{v^{2}}{\pi^{3} k} \int_{0}^{\infty} d x \frac{a x \sin k x}{\left(a^{2}+x^{2}\right)^{3}}
$$

The $k^{2}$ term is

$$
\frac{d I_{0}}{d k^{2}} \underset{k \rightarrow 0}{\longrightarrow}-\frac{v^{2}}{32 \pi^{2}}
$$

Note that this is a finite result, with no $\ln (k a)$ dependence. Thus there is no renormalization (in the scaling sense) of the $|\nabla \Phi|^{2}$ term.

We now consider possible renormalization of the $\left|\partial_{\tau} \Phi\right|^{2}$ term. It is sufficient in this case to compute $I^{\prime}=$ $\partial I /\left.\partial \omega^{2}\right|_{\omega=0}$ for small $k$. A divergent (scaling) renormalization should appear in $I^{\prime}$ again as a $\ln (k a)$, so $k$ 
must be kept non-zero until the end of the calculation. Differentiating Eq. (B1) gives

$$
\begin{aligned}
I^{\prime}= & v^{2} \int_{\boldsymbol{p}_{1}, \boldsymbol{p}_{2}, \nu}\left[1-\frac{v^{2} p_{1}^{2}}{\nu^{2}+v^{2} p_{1}^{2}}\right]\left[\frac{3}{\left(\nu^{2}+p_{2}^{2}\right)^{2}}\right. \\
& \left.-\frac{4 p_{2}^{2}}{\left(\nu^{2}+p_{2}^{2}\right)^{3}}\right] e^{-p_{1} a-p_{2} a}(2 \pi)^{3} \delta\left(\boldsymbol{p}_{1}+\boldsymbol{p}_{2}+\boldsymbol{k}\right) .
\end{aligned}
$$

It is convenient to take the $v \rightarrow 0$ limit in the integrand at this stage, which allows one to drop the second term in the first set of square brackets in Eq. (B7). Performing the frequency integrals then gives

$$
\begin{aligned}
I^{\prime} & =v^{2} \int_{\boldsymbol{p}_{1}, \boldsymbol{p}_{2}}\left[\frac{3}{4 p_{2}^{3}}-4 p_{2}^{2} \frac{3}{16 p_{2}^{5}}\right] \\
& \times e^{-p_{1} a-p_{2} a}(2 \pi)^{3} \delta\left(\boldsymbol{p}_{1}+\boldsymbol{p}_{2}+\boldsymbol{k}\right),
\end{aligned}
$$

which is identically zero! The $\left|\partial_{\tau} \Phi\right|^{2}$ term is therefore unrenormalized.

\section{APPENDIX C: PERTURBATION THEORY FOR THE VERTEX FUNCTIONS}

This appendix comprises the Feynman diagrams to one-loop order for the effective free energy functional of the supersolid to superfluid transition. We start with a list of the contributions to one-loop order to the fully wavevector- and frequency-dependent two-point vertex functions. The Feynman diagrams for the vertex functions $\Gamma_{l n}$, with $l$ fields of the superfluid phase and $n$ Ising fields, are depicted in Figs. (13)-(16). The corresponding analytical expressions for $\Gamma_{02}(\mathbf{q}, \omega)$ is given by

$$
\Gamma_{02}^{(a)}(\mathbf{q}=0, \omega=0)=\frac{u}{2} \int_{\mathbf{p}, \mu} \frac{1}{t+(\mu / c)^{2}+p^{2}},
$$

and

$$
\begin{gathered}
\Gamma_{02}^{(b)}(\mathbf{q}, \omega)=4(\sigma c v)^{2} \int_{\mathbf{p}, \mu} \frac{1}{(\omega+\mu)^{2}+c^{2}\left[t+(\mathbf{p}+\mathbf{q})^{2}\right]} \\
\times \frac{\mu^{2}}{\left[\mu^{2}+v^{2} p^{2}\right]}
\end{gathered}
$$

Performing the frequency integrals using the residue theorem we find

$$
\Gamma_{02}^{(a)}(\mathbf{q}=0, \omega=0)=-t \frac{u c}{8} \frac{S_{d}}{\varepsilon}
$$

where we have subtracted the $T_{c}-$ shift $\left.\Gamma_{02}^{(a)}(\mathbf{0}, 0)\right|_{0}$ and

$$
\begin{gathered}
\Gamma_{02}^{(b)}(\mathbf{q}, \omega)=\frac{4(\sigma v c)^{2}}{c} \int_{\mathbf{p}}\left\{\frac{1}{2 A}-\left(\frac{v}{c}\right)^{2}(\mathbf{p}+\mathbf{q})^{2} \frac{A+B}{2 A B}\right. \\
\left.\times \frac{1}{(\omega / c)^{2}+(A+B)^{2}}\right\},
\end{gathered}
$$

where $A=\sqrt{t+p^{2}}$ and $B=(v / c) \sqrt{(\mathbf{p}+\mathbf{q})^{2}}$. The wavevector integral of Eq. (C4) can be evaluated for several limits listed below:

(a) $\omega=0, \mathbf{q}=\mathbf{0}, t \neq 0$ :

$$
\Gamma_{02}^{(b)}(\mathbf{0}, 0)=-t(v \sigma)^{2} c \frac{S_{d}}{\varepsilon} \frac{1}{(1+v / c)^{2}},
$$

where we have again subtracted the corresponding $T_{c}-$ shift $\left.\Gamma_{02}^{(b)}(\mathbf{0}, 0)\right|_{0}$.

(b) $\omega=0, \mathbf{q} \neq \mathbf{0}, t=0$ :

$$
\Gamma_{02}^{(b)}(\mathbf{q}, 0)=-q^{2} v^{3} \sigma^{2} \frac{S_{d}}{\varepsilon} \frac{1}{(1+v / c)^{3}} \mathcal{A}(v / c, d),
$$

with

$$
\mathcal{A}(v / c, d)=\frac{8-2 d+(v / c)(9-3 d)+(v / c)^{2}(3-d)}{d}
$$

(c) $\omega \neq 0, \mathbf{q}=\mathbf{0}, r=0$ :

$$
\Gamma_{02}^{(b)}(\mathbf{q}, 0)=\left(\frac{\omega}{c}\right)^{2} 2 v^{3} \sigma^{2} \frac{S_{d}}{\varepsilon} \frac{1}{(1+v / c)^{3}} .
$$

The analytical expression for $\Gamma_{20}(\mathbf{q}, \omega)$ at $\omega=0$ reads

$$
\Gamma_{20}(\mathbf{q}, 0)=2 \omega^{2} \sigma^{2} c \int_{\mathbf{p}, \mu} \frac{1}{\left[t+\mu^{2}+p^{2}\right]^{2}},
$$

and after performing the frequency integrals using the residue theorem we find

$$
\Gamma_{20}(\mathbf{q}, 0)=\frac{\omega^{2} c}{2} \sigma^{2} \frac{S_{d}}{\varepsilon} .
$$

The vertex function $\Gamma_{12}(\mathbf{q}, 0):=\Gamma_{12}(\mathbf{q}, \omega ;-\mathbf{q} / 2,0 ;-\mathbf{q} / 2,0)$ to one-loop order has two contributions

$$
\Gamma_{12}^{(a)}(\mathbf{q}, 0)=-\omega \sigma u \int_{\mathbf{p}, \mu} \frac{1}{\left[t+(\mu / c)^{2}+p^{2}\right]^{2}},
$$

and

$$
\Gamma_{12}^{(b)}(\mathbf{q}, 0)=-8 \omega \sigma^{3} \int_{\mathbf{p}, \mu} \frac{\mu^{2}}{\left[t+(\mu / c)^{2}+p^{2}\right]^{2}\left[(\mu / v)^{2}+p^{2}\right]} .
$$

After performing the frequency integrals we find

$$
\begin{gathered}
\Gamma_{12}^{(a)}(\mathbf{q}, 0)=-\omega \sigma u c \frac{S_{d}}{\varepsilon} \\
\Gamma_{12}^{(b)}(\mathbf{q}, 0)=-2 c \omega \sigma^{3} v^{2} \frac{S_{d}}{\varepsilon} \frac{1}{(1+v / c)^{2}}
\end{gathered}
$$

The vertex function $\Gamma_{04}$ at zero external frequencies and momenta has three contributions. 


$$
\begin{gathered}
\Gamma_{04}^{(a)}=-\frac{3}{2} u^{2} c \int_{\mathbf{p}, \mu} \frac{1}{\left[t+(\mu / c)^{2}+p^{2}\right]^{2}}, \\
\Gamma_{04}^{(b)}=-24 u \sigma^{2} \int_{\mathbf{p}, \mu} \frac{\mu^{2}}{\left[t+(\mu / c)^{2}+p^{2}\right]^{2}\left[(\mu / v)^{2}+p^{2}\right]}, \\
\Gamma_{04}^{(c)}=-96 \sigma^{4} \int_{\mathbf{p}, \mu} \frac{\mu^{4}}{\left[t+(\mu / c)^{2}+p^{2}\right]^{2}\left[(\mu / v)^{2}+p^{2}\right]^{2}} .
\end{gathered}
$$

After performing the frequency integrals we find

$$
\begin{aligned}
\Gamma_{04}^{(a)} & =-\frac{3 u^{2} c}{8} \frac{S_{d}}{\varepsilon} \\
\Gamma_{04}^{(b)} & =-6 u c(\sigma v)^{2} \frac{S_{d}}{\varepsilon} \frac{1}{(1+v / c)^{2}}, \\
\Gamma_{04}^{(c)} & =-24(\sigma v)^{4} c \frac{S_{d}}{\varepsilon} \frac{1}{(1+v / c)^{3}} .
\end{aligned}
$$

[1] P.W. Anderson, Phys. Rev. 115, 2 (1959).

[2] N.F. Mott, Proc. Phys. Soc. London Ser. A62, 416 (1949).

[3] For a review see e.g.: D. Belitz and T.R. Kirkpatrick, Rev. Mod. Phys. 66, 261 (1994).

[4] See e.g.: M.P.A. Fisher, P.B. Weichman, G. Grinstein, and D.S. Fisher, Phys. Rev. B 40, 546 (1989); and references cited therein.

[5] C. Bruder, R. Fazio, and G. Schön, Phys. Rev. B 47, 342 (1993); C. Bruder and G. Schön, in KT Transition and Superconducting Arrays, edited by D. Kim et al., (Min Eum Sa Co., Seoul, 1993), p. 175.

[6] E. Roddick and D.H. Stroud, Phys. Rev. B 48, 16600 (1993).

[7] K-H. Wagenblast, Diplomarbeit Universität Karlsruhe 1994, unpublished.

[8] A. van Otterlo, $\mathrm{PhD}$ Thesis, Universität Karlsruhe (1994).

[9] A. van Otterlo, and K.-H. Wagenblast, Phys. Rev. Lett. 72, 3598 (1994).

[10] G.G. Batrouni, R.T. Scalettar, G.T. Zimanyi, and A.P. Kampf, Phys. Rev. Lett. 74, 2527 (1995); R.T. Scalettar, G.G. Batrouni, A.P. Kampf, and G.T. Zimanyi, Phys. Rev. B 51, 8467 (1995).

[11] A.P. Kampf, G.T. Zimanyi, G.G. Batrouni, and R.T. Scalettar, unpublished (1995).

[12] A. van Otterlo, K.-H. Wagenblast, R. Baltin, C. Bruder, R. Fazio, and G. Schön, Phys. Rev. B 52, 16176 (1995).

[13] C. Bruder, R. Fazio, A.P. Kampf, A. van Otterlo, and G. Schön, Physica Scripta T 42, 159 (1992).
[14] H. Matsuda, and T. Tsuneto, Suppl. Prog. Theor. Phys. 46, 411 (1970).

[15] K. Liu, and M. Fisher, J. Low. Temp. Phys. 10, 655 (1973).

[16] G. G. Batrouni, R. T. Scalettar, and G. T. Zimanyi, Phys. Rev. Lett. 65, 1765 (1990); R. T. Scalettar, G. G. Batrouni, and G. T. Zimanyi, Phys. Rev. Lett. 66, 3144 (1991).

[17] P. Niyaz, C. Y. Fong, R. T. Scalettar, and G. G. Batrouni, Phys. Rev. B 50, 362 (1994); P. Niyaz, R. T. Scalettar, C. Y. Fong, and G. G. Batrouni, Phys. Rev. B 44, 7143 (1991).

[18] W. Krauth and N. Trivedi, Europhys. Lett. 14, 627 (1991).

[19] E.S. Sørensen, M. Wallin, S.M. Girvin and A.P. Young, Phys. Rev. Lett 69, 828 (1992); M. Wallin, E.S. Sørensen, S.M. Girvin, and A.P. Young, Phys. Rev. B 49, 12115 (1994).

[20] E. Frey, D.R. Nelson, and D.S. Fisher, Phys. Rev. B 49, 9723 (1994).

[21] L. Balents, and D.R. Nelson, Phys. Rev. Lett. 73, 2618 (1994); Phys. Rev. B 52, 12951 (1995).

[22] B.I. Halperin, T.C. Lubensky, S.-K. Ma, Phys. Rev. Lett. 32, 292 (1974); C. Dasgupta and B.I. Halperin, Phys. Rev. Lett. 47, 1556 (1981); L. Radzihovsky, Euro. Phys. Lett. 29, 227 (1995).

[23] See, e.g., L.B. Ioffe and A.J. Millis, Phys. Rev. B 51, 16151 (1995); and references therein.

[24] D.J. Bergman and B.I. Halperin, Phys. Rev. B 13, 2145 (1976); and references cited therein.

[25] M.A. de Moura, T.C. Lubensky, Y. Imry, and A. Aharony, Phys. Rev. B 13, 2176 (1976).

[26] T. Nattermann, J. Phys. C 10, 1757 (1977).

[27] D.J. Wallace, J. Phys. C 6, 1390 (1973).

[28] A. Aharony, Phys. Rev. B 8, 3349, (1973);8, 3358 (1973); 8, 4270 (1973); Phys. Rev. Lett. 31, 1494 (1973).

[29] I.J. Ketley and D.J. Wallace, J. Phys. A 6, 1667 (1973).

[30] E. Brézin, J.C. Guillou, and J. Zinn-Justin, Phys. Rev. B 10, 892 (1974).

[31] T. Nattermann and S. Trimper, J. Phys. A 8, 2000 (1975).

[32] I.F. Lyuksyutov and V.L. Pokrovsky, Pis'ma Zh. Teor. Fiz. 21, 22 (1975) [JETP Lett. 21, 9 (1975)]; I.F. Lyuksyutov, Phys. Lett. 56A, 135 (1976).

[33] J. Rudnick,Phys. Rev. B 18, 1406 (1978).

[34] H. H. Jacobson and D. J. Amit, Ann. Phys. (N.Y.) 133, 57 (1981).

[35] See A. Aharony et al., Phys. Rev. Lett. 57, 1012 (1986); and references therein.

[36] A dangerous irrelevant perturbation is one which is irrelevant at the critical fixed point, but relevant at the ordered fixed point, and often leads to violations of hyperscaling.

[37] U.C. Täuber and F. Schwabl, Phys. Rev. B 48, 186 (1993).

[38] D. J. Amit, Field Theory, the Renormalization Group, and Critical Phenomena, revised 2nd ed. (World Scientific, Singapore, 1993); J. Zinn-Justin, Quantum Field Theory and Critical Phenomena, 2nd ed. (Clarendon Press, Oxford, 1993). 
[39] G. t'Hooft and M. Veltman, Nucl. Phys. B 44, 189 (1972).

[40] G. Zimanyi, private communication. 
TABLE I. Transformation properties of the checkerboard $\Phi$ and collinear order parameter $\Psi$ under coordinate transformations.

\begin{tabular}{ccc}
\hline \hline Symmetry & Coord. Transf. & Field Transf. \\
\hline Translation & $x_{i} \rightarrow x_{i}+1$ & $\Phi \rightarrow-\Phi$ \\
& & $\psi_{i} \rightarrow-\psi_{i}$ \\
Reflection & $x_{i} \rightarrow-x_{i}$ & Invariant \\
$\pi / 2$ Rotation & $x_{i} \rightarrow x_{j}$ & $\psi_{i} \leftrightarrow \psi_{j}$ \\
Time Reversal & $x_{j} \rightarrow-x_{i}$ & \\
& $\tau \rightarrow-\tau$ & $\theta \rightarrow-\theta$ \\
\hline \hline
\end{tabular}




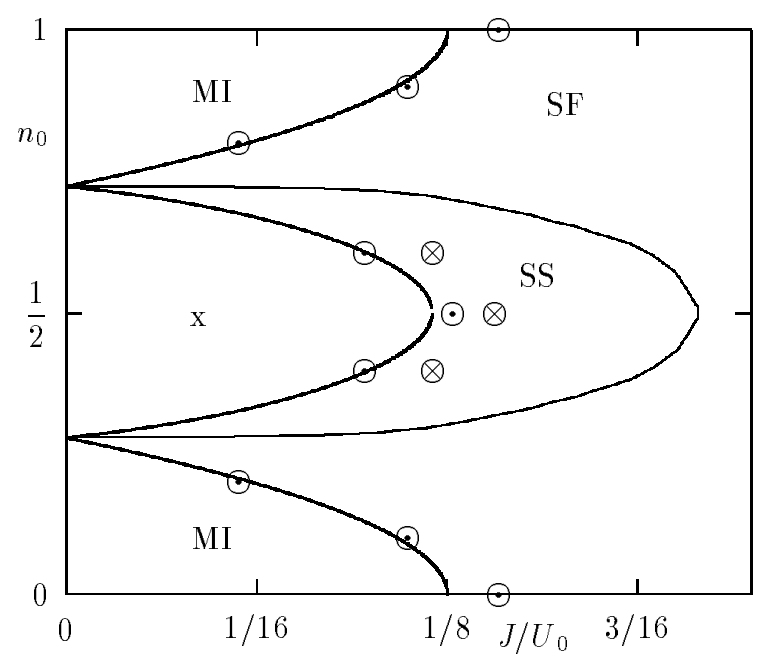

FIG. 1. Phase diagrams for soft-core bosons, as obtained from the mean-field analysis 6, 17 of the Quantum Phase Hamiltonian with on-site and nearest-neighbor $\left(U_{1} / U_{0}=1 / 5\right)$ interaction, $n_{0}=\mu / \sum_{i} U_{i j}$. The symbols are the Monte Carlo data as discussed in Ref. [9, 12. The checkerboard charge-density wave is denoted by "X", the supersolid phase by "SS", the superfluid phase by "SF" and the Mott-insulating phase by "MI". Taken from Ref. [12].

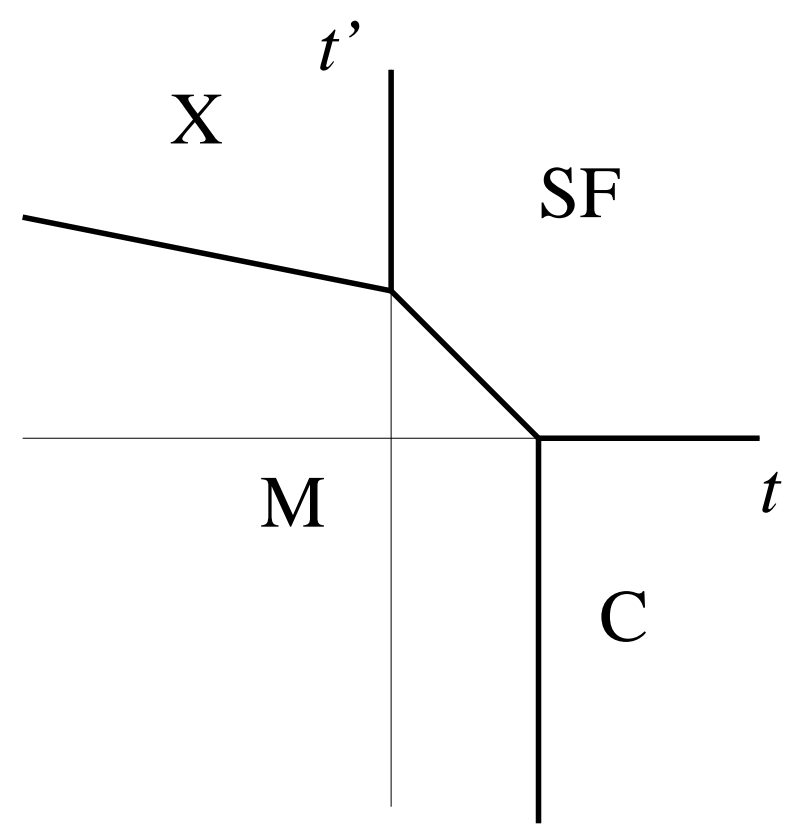

FIG. 2. Topology of the mean-field phase diagram.

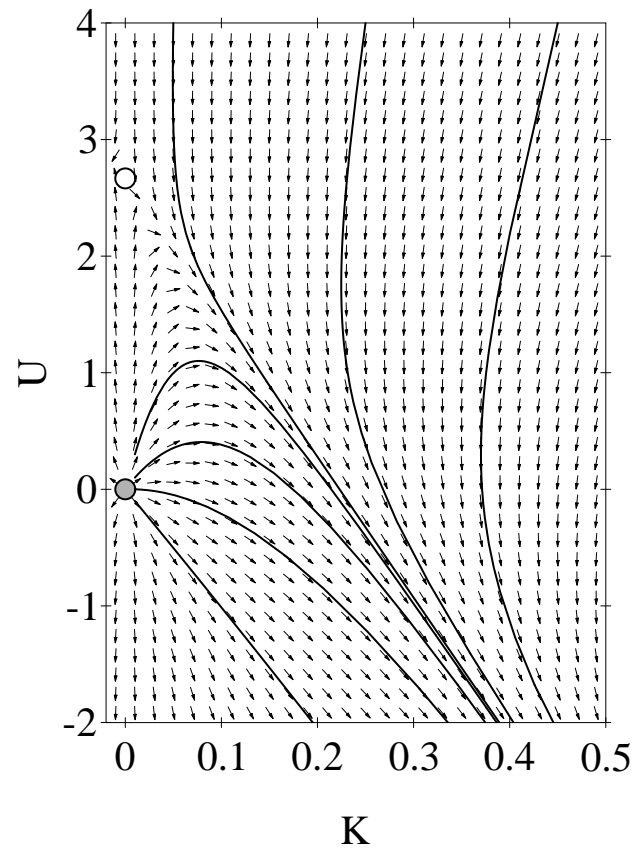

FIG. 3. Momentum-shell flow diagram at the SF-X critical point for $V=0$ in $d=2$ dimensions. The Ising I fixed point and the Gaussian I fixed point are indicated by the open and shaded circle, respectively.

(a)

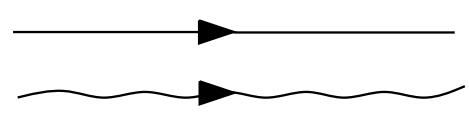

(b)

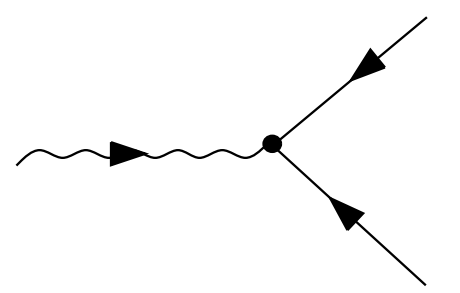

(c)

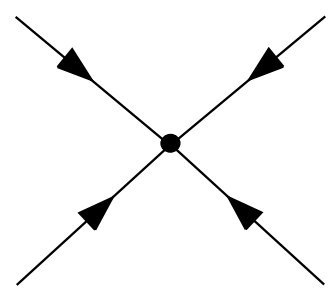

FIG. 4. Elements of perturbation theory. (a) Propagators for the Ising field $\Phi$ (solid line) and the superfluid phase $\Theta$ (wiggly line). (b) Vertex for the coupling between the superfluid phase and the crystalline order parameter. (c) $\Phi^{4}$ term for the Ising field $\Phi$. 


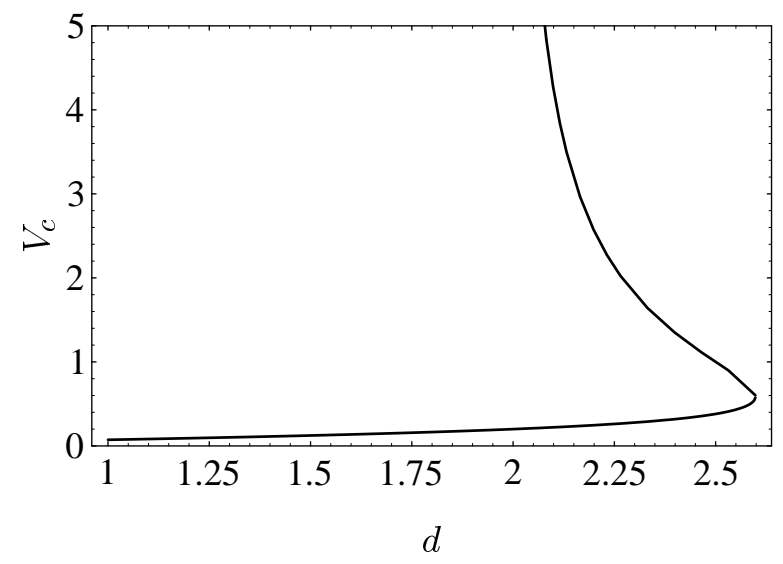

FIG. 5. Critical values $V_{c}^{1,2}$ (lower and upper curve) for the ratio $V=v / c$ as a function of the dimension $d$. For $V<V_{c}^{1}$ the flow of $V$ tends to $V=0$ and one gets runaway trajectories for the flow of the coupling constant. For $V_{c}^{2}>V>V_{c}^{1}$ the flow of $V$ tends to $V_{c}^{2}$, and finally for $V>V_{c}^{2}$ the flow of $V$ tends to $V=\infty$.

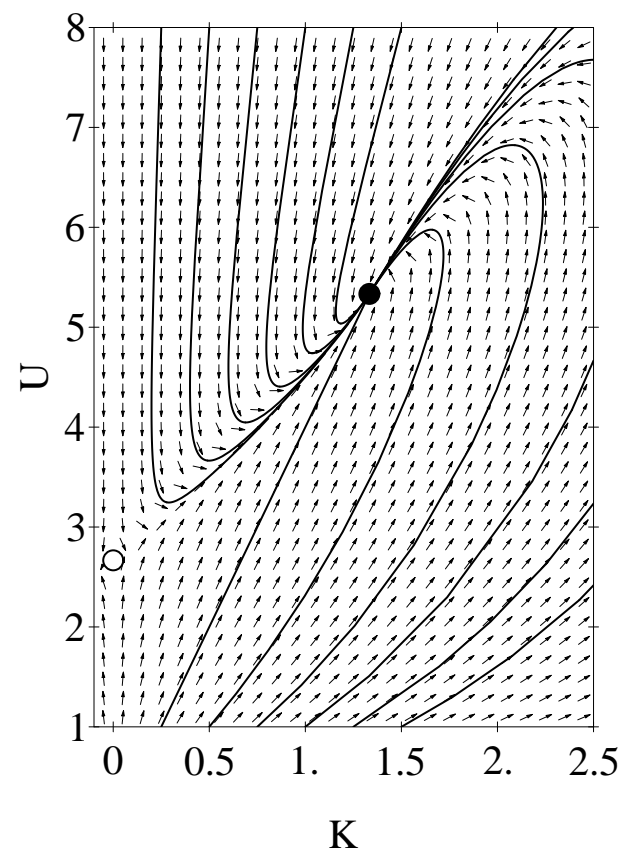

FIG. 6. Flow diagram of the SF-X transition in the limit $V=\infty$ for $d=2$. The topology of the flow is determined by the presence of the unstable Ising fixed point at $(K, U)=(0,8 / 3)$ (open circle) and the stable non-Bose liquid fixed point at $(K, U)=(4 / 3,16 / 3)$ (filled circle).
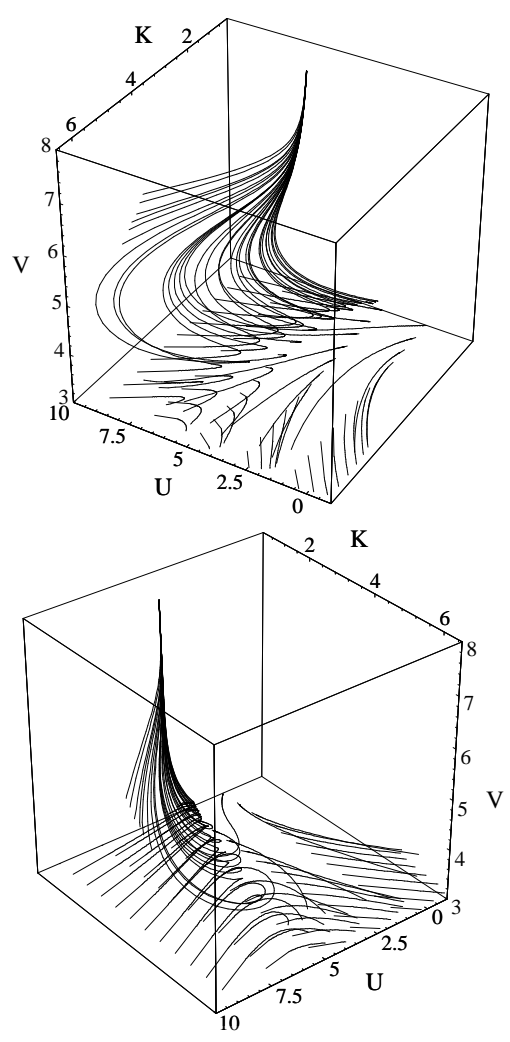

FIG. 7. Two different views of the RG-trajectories from the RG-equations Eqs. (7.38-7.40).
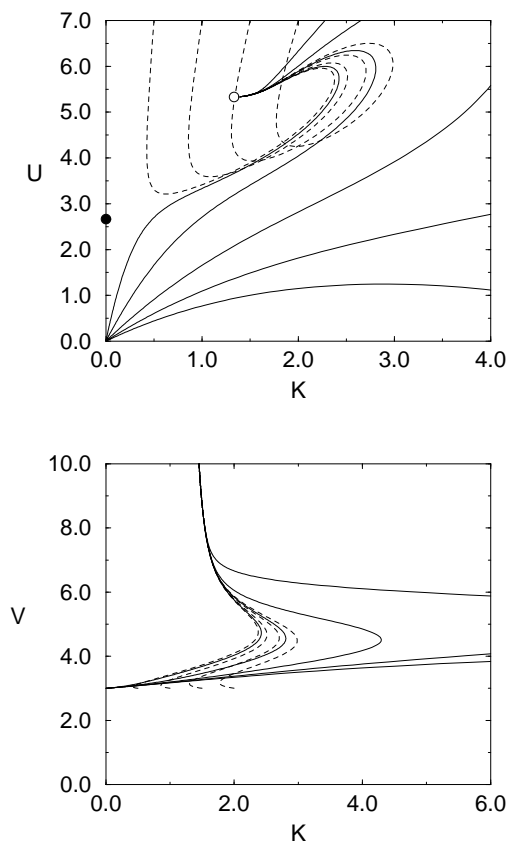
FIG. 8. Two-dimensional projections of the three--dimensional $(U, K, V)$-flow diagram. The trajectories all start at $V=3$ and a set of values for $U$ and $K$. The first set (dashed lines) is $U=7$ and $K=0.5,1.0,1.5,2.0$, the second set (solid lines) is $U=0.01$ and $K=0.001,0.005,0.0075,0.01$.
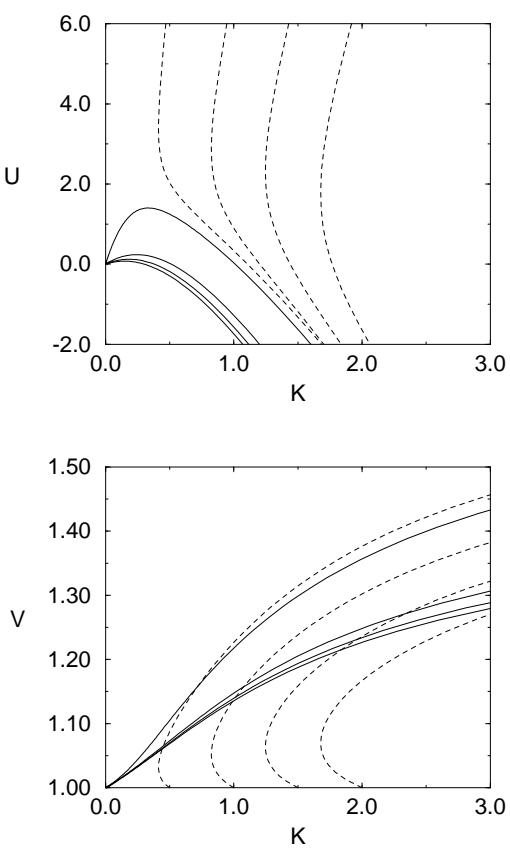

FIG. 9. Two-dimensional projections of the three--dimensional $(U, K, V)$-flow diagram. The trajectories all start at $V=1$ and a set of values for $U$ and $K$. The first set (dashed lines) is $U=7$ and $K=0.5,1.0,1.5,2.0$, the second set (solid lines) is $U=0.01$ and $K=0.001,0.005,0.0075,0.01$.

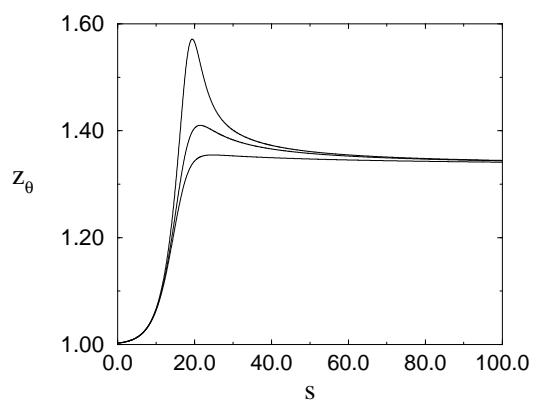

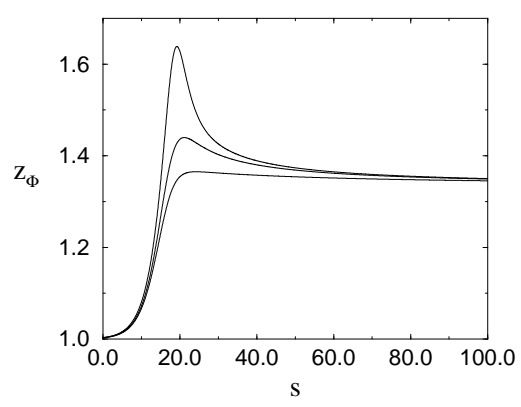

FIG. 10. Effective dynamic critical exponents a) $z_{\Theta}(s)$ and b) $z_{\Phi}$ for the initial values $U(s=1)=8 / 3, K(s=1)=1 / 100$ and a series of rations of sound velocities $V(1)=10^{-k}$ with $k=3,2, \ldots,-1$.

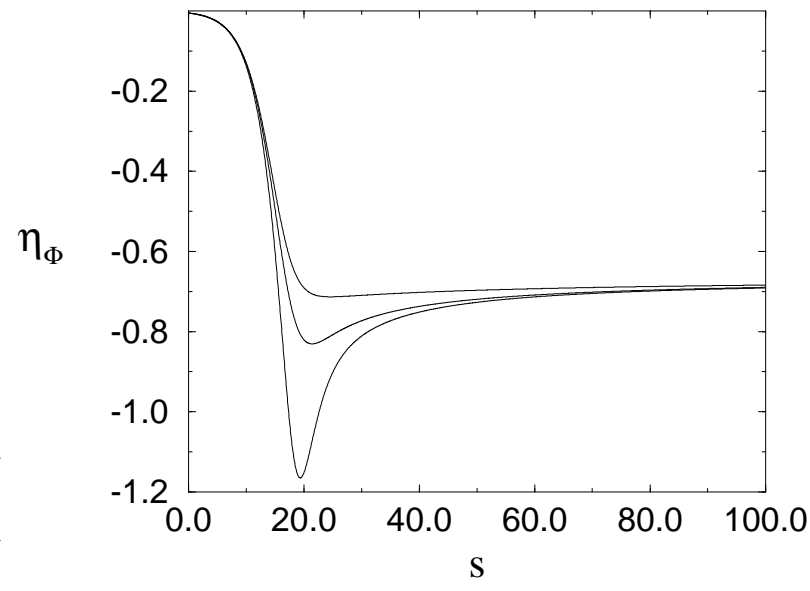

FIG. 11. Effective Fisher exponent $\eta_{\Phi}(s)$ for the initial values $U(s=1)=8 / 3, K(s=1)=1 / 100$ and a series of rations of sound velocities $V(1)=10^{-k}$ with $k=3,2, \ldots,-1$.

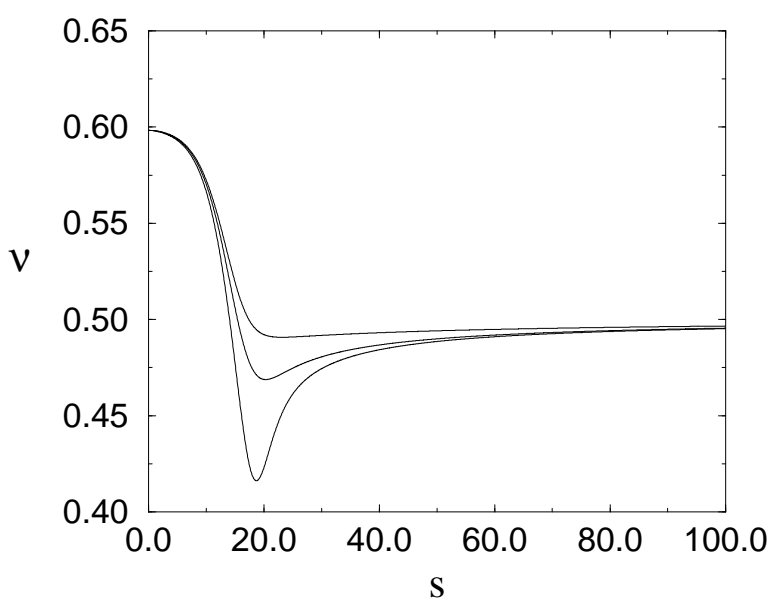


FIG. 12. Effective correlation length exponent $\nu(s)$ for the initial values $U(s=1)=8 / 3, K(s=1)=1 / 100$ and a series of ratios of sound velocities $V(1)=10^{-k}$ with $k=3,2, \ldots,-1$. 

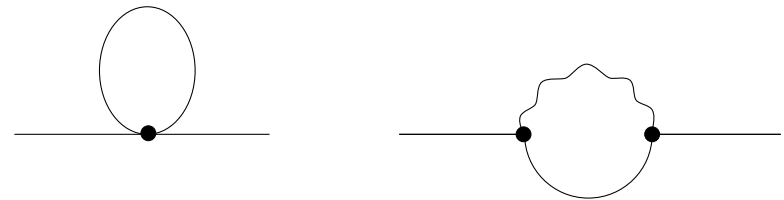

FIG. 13. Feynman diagrams for the dynamic perturbation expansion of $\Gamma_{02}$ to one-loop order.

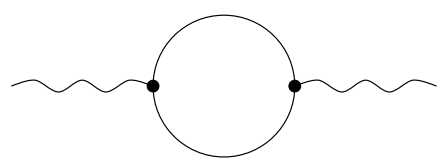

FIG. 14. Feynman diagrams for the dynamic perturbation expansion of $\Gamma_{20}$ to one-loop order.
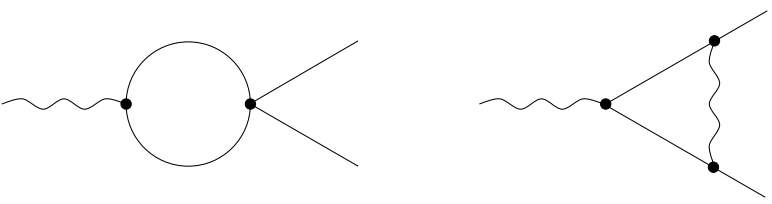

FIG. 15. Feynman diagrams for the dynamic perturbation expansion of $\Gamma_{12}$ to one-loop order.
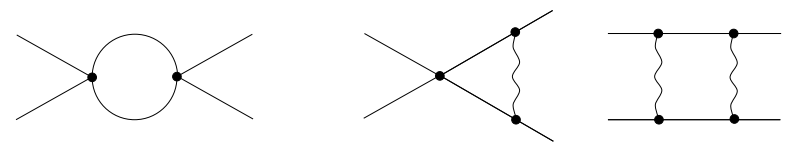

FIG. 16. Feynman diagrams for the dynamic perturbation expansion of $\Gamma_{04}$ to one-loop order. 